\title{
Exploring the Direction on the Environmental and Business Performance Relationship at the Firm Level. Lessons from a Literature Review
}

\author{
Anna Mazzi ${ }^{1}$, Sara Toniolo ${ }^{1}$, Alessandro Manzardo ${ }^{1}$, Jingzheng Ren ${ }^{2}$ and Antonio Scipioni ${ }^{1, *}$ \\ 1 Department of Industrial Engineering, University of Padova, Via Marzolo 9, 35131 Padova, Italy; \\ anna.mazzi@unipd.it (A.M.); sara.toniolo@unipd.it (S.T.); alessandro.manzardo@unipd.it (A.M.) \\ 2 Department of Technology and Innovation, University of Southern Denmark, Campusvej 55, \\ 5230 Odense M, Denmark; jire@iti.sdu.dk \\ * Correspondence: scipioni@unipd.it; Tel.: +39-049-827-5539; Fax: +39-049-827-5785
}

Academic Editor: Domingo Ribeiro-Soriano

Received: 4 October 2016; Accepted: 16 November 2016; Published: 19 November 2016

\begin{abstract}
The interest of scientists and companies in understanding the business implications of environmental investment is timely; however, a dilemma remains at the firm level: is the environment a "strategic competitive factor", as in the "Porter point of view", or is it a "luxury good", as in the "Wagner point of view"? Our research contributes to this debate through a review of the papers published in scientific journals between 2000 and 2015 that discussed the direction of the relationship between the environmental and business performances of enterprises. The objectives of the research are: (a) to verify if there is an agreement in the scientific literature of the last 15 years about the "Porter-Wagner dilemma" when focusing at the firm level; (b) to underline the prevalent cause and effect directions of the relationship between environmental and business performance; and (c) to investigate the reasons for any disagreements in this topic among the scientists. The results show that the main agreement regards the positive bi-directional relationship, as a virtuous cyclic approach with mutual effects between business and environmental performance; nevertheless, more complex hypotheses emerge, such as nonlinear and/or conditional relationship, that need to be further explored. On the other hand, the Porter-Wagner dilemma remains, and the main reason for the non-agreement among scientists can be due to the several non-homogeneous variables considered in the analyses. Thereafter, as lesson for scientists, the priority is to share univocal methods to measure firms' environmental and business performances.
\end{abstract}

Keywords: business performance; enterprises; environmental performance; literature review; Porter-Wagner dilemma

\section{Introduction}

The issue of environmental sustainability is of great interest for companies today [1,2]. The international community encourages companies to adopt cleaner production systems and technologies [3-5] and to understand the business implications of environmental investment [6-9]. The market seems to reward environmentally responsible organizations, and many companies around the world are increasingly interested in environmental issues and are introducing them as strategic variables in their businesses (see also [10-16]). Increasing environmental certifications and labeling can be understood to demonstrate that environmental improvement benefits firms' performance (see also [17-22]). On the other hand, we must recognize that companies with better economic/financial performance are often able to promote environmental initiatives and/or adopt cleaner production technologies (see also [23-26]). 
The so-called "neoclassical" approach that considers the environment as a negative externality and focuses on the environment in terms of compliance with regulatory requirements is considered to be outdated [27-33].

The dilemma thus arises: is it beneficial for a company to invest in the environment, or is environmental commitment achievable only for enterprises with good economic performance? In other words, is the environment a "strategic competitive factor", such as in the "Porter point of view" [34-36], or is it a "luxury good", such as in the "Wagner point of view" [24,26]?

There is a longstanding debate regarding this topic, "like finding the Holy Grail" [37]. Nevertheless, the growing body of studies is often inconclusive in solving the "Porter-Wagner dilemma": does better environmental performance determine better business performance of companies or vice versa (see also [38-41])? Now, we are aware that the answer to this dilemma has important implications for business strategies and institutional policies (see also [30,42,43]).

Above all, the analysis of the relationship between environmental performance (EP) and business performance (BP) is even more relevant from the firms' perspective, since companies have great interest in learning about the economic benefits of environmental responsibility [44,45]. On the other hand, at the firm's level, the difficulty of quantifying the economic added value of the adoption of environmental strategies remains [46,47].

This paper contributes to this debate, through a comprehensive review of the scientific literature of the last 15 years, with the following objectives: (a) to verify if there is an agreement about the "Porter-Wagner dilemma" when focusing at the firm level; (b) to underline the prevalent cause and effect directions of the relationship between EP and BP of companies; and (c) to investigate the reasons for any disagreements in this topic among the scientists.

In this paper initially we summarize the literature background and underline the main literature gaps concerning the "Porter-Wagner dilemma". Coherently with these gaps, in Section 3 we define the research theoretical framework, including research questions and hypotheses, and the materials and methods adopted to conduct the systematic literature review. Hence, in Section 4, we summarize the research results, and we compare them among the research hypotheses. In Section 5, we discuss these results with the research questions. Finally, in Section 6, we conclude the paper with notable statements and future perspectives, both for managers, practitioners, and scientists.

\section{Background}

Many authors have extensively debated the relationship between EP and BP, both in empirical and theoretical studies, starting with the awareness of the complexity of the topic [48,49].

At the empirical level, the question "does it pay to be green" has been discussed by many authors across different disciplines, which has led to conflicting results, as pointed up in relevant literature reviews. Molina-Azorín et al. [50], in analyzing 32 quantitative studies, noted the different variables adopted by the authors, the different sectors and countries considered, and the different findings obtained. Horváthová [51], with a meta-regression analysis of 37 empirical studies, proposed a summary of the main reasons for the inconclusive results: the firm size, country location, environmental criteria and time coverage of the analyses were considerable factors that influenced the variation in empirical evidence. Dixon-Fowler et al. [52], according to a meta-analytical review of 71 papers concerning corporate environmental performance and corporate financial performance, focused on proactive and reactive environmental assets: they drew on some inconsistencies from the previous research, such as the inappropriate choice of variables and the different methodologies adopted to support the analysis. This lack was confirmed by the literature review of Guenther and Hoppe [53]. Albertini [54], with a meta-analysis of 52 studies, partially confirmed and partially extended this discussion: they revealed that environmental and financial indexes, regional differences, activity sectors and run time are the main moderator factors in the EP-BP relationship. Goyal et al. [55], according to a review of 101 research papers, demonstrated that the discussion about corporate sustainability performance and firm performance is still active and that feedback from previous 
studies was not universally consistent about the direction of the relationship. Endrikat et al. [56], with a meta-analytic review of 149 studies, explored the direction of causality in the EP-BP link and demonstrated a positive and partially bi-directional relationship in proactive rather than reactive environmental strategies; however, they underlined the inconsistency of some previous studies.

From a theoretical point of view, notable studies gave convincing answers to the "Porter Wagner-dilemma". Lankoski [57] proposed an integrative synthesis of knowledge of the relationship between corporate responsibility activities and economic performance and pointed out that different corporate responsibility activities produce different economic output. Potts et al. [58] advanced the "co-evolutionary" model in which the dynamics of economic and ecological systems are connected by the business behavior. Orlitzky [59], with a comprehensive literature review of corporate social responsibility, discussed how different disciplines influenced different empirical results in the relationship between environmental, social and financial performance. Boons et al. [60] summarized the conceptual discussion in the research agenda of sustainable innovation and business models, emphasizing that environmental innovation supports business performance. Dües et al. [14] provided evidence that lean supply chain management practices benefit environmental performance and, in turn, green practices influence lean business. Van der Byl and Slawinski [61], with a comprehensive review of research on corporate sustainability, outlined previous studies' approaches of demonstrating the existence of a relationship between corporate environmental and social responsibility and firm performance and confirmed that the methodological approach of studying this relationship is still evolving.

From this overview, some significant elements emerge. Many authors confirm the existence of a relationship between EP and BP, although in some cases this relationship has not been strongly verified. Moreover, some authors underline the difficulty of verifying this relationship. Finally, even if a relationship between EP and BP exists, it is not clear what the direction of the relationship is. A new study to overcome some limitations of previous reviews is useful. First of all, the focus of the study must be on the companies, in all sectors and dimensions and worldwide. Then, it is important to analyze the papers that discuss the direction of the relationship, not simply the existence of the relationship. Moreover, it is necessary to analyze the papers assuming various possible types of cause and effect relationships, not only linear and not only one-way. Finally, it is relevant to compare the conclusions of the papers in order to underline what the prevalent cause and effect directions of the EP-BP relationship are, and to investigate the reasons for any disagreements among the studies concerning the different types of relationships.

To fill these literature gaps, the objective of this research is to determine whether there is agreement in the papers published in scientific journals regarding the direction of the relationship between the EP and the BP, focusing on enterprises. Consistent with this research objective, our research questions are: Is there a consensus in scientific papers on the direction of the relationship between EP and BP at the firm level? If so, what are the prevalent directions? If not, what are the reasons that do not lead the studies to a consensus view?

\section{Research Hypotheses and Methodology}

\subsection{Research Hypotheses}

To answer the research questions, we formulated four sub-questions: (i) Which recent papers address the relationship between the EP and BP of enterprises? (ii) Is there a consensus among the authors to declare the existence of an EP-BP relationship? (iii) Is there a specific direction of the EP-BP relationship? (iv) Are there some reasons do not lead the studies to a consensus view in the direction of EP-BP relationship?

To analyze sub-question (i), we consider all of the papers published from 2000 to 2015 in the scientific journals available on web platforms that discuss the EP-BP relationship (in line with $[51,56]$ ). 
To analyze sub-question (ii), we consider two alternative answers, corresponding to the following research hypotheses (Figure 1):

HO: There is a relationship between EP and BP of enterprises

H1: There is no relationship between EP and BP of enterprises

To analyze sub-questions (iii) and (iv), we allow hypothesis $\mathrm{H} 0$ and formalize five different types of relationships between EP and BP that are not necessary mutually exclusive (Figure 1) (in line with $[37,41,53,56,62-64])$.

H0.1: Better EP determines better BP (in accordance to the "Porter point of view")

H0.2: Worse EP determines worse BP (as a "negative Porter point of view")

H0.3: Better BP determines better EP (in accordance to the "Wagner point of view")

H0.4: Worse BP determines worse EP (as a "negative Wagner point of view")

H0.x: There is another possible EP-BP relationship (e.g., inverse, non-linear, $U$-shaped, and conditional)

\subsection{Research Methodology}

To verify the research hypotheses, we conducted qualitative research based on a systematic literature review, in line with Luederitz et al. [65], exploring the research topic in scientific papers published during the years specified. As synthesized in Table 1, the research was conducted in four steps, each one corresponding to a specific sub-question.

Step (I) To verify "which recent papers address the relationship between EP and BP at the firm level" (Table 1, sub-question (i)), a bibliographical survey was conducted with international databases (ISI Web of Knowledge and the main editors' libraries) using specific research keywords (as "environmental performance", "business performance", "firms", and "relationship" and their synonyms) (coherently with us, see also $[51,52,56,66,67])$. The time-period considered for this survey starts from 2000, when the scientific debate started (in line with $[30,38,61,68]$ ). In order to include in the literature analysis all the relevant papers, we selected them in two steps, coherently with the suggestions of Luederitz et al. [66]: (I-A) Data Screening, which concerns the search in the established databases through the established keywords; and (I-B) Data Cleaning, which concerns the evaluation of each papers selected in the previous (I-A) step, in order to decide their inclusion in the research sample, based on the coherency of the title, abstract and full text with the research sub-question (i).

Then, a descriptive analysis of the selected papers was conducted in terms of country, industrial sector, type of research, sample of companies, research goals, business and environmental variables considered to measure firms performance and research conclusions (in line with $[37,50,65,67,69]$ ).

In order to confirm or deny the existence of an EP-BP relationship (see Table 1, sub-question (ii)), we analyzed results, discussion and conclusions in all of the selected papers, verifying our two research hypotheses ( $\mathrm{H} 0$ and $\mathrm{H} 1$ ) (similar to us, see also [51,66,70]).

In order to verify the direction of the EP-BP relationship (see Table 1, sub-question (iii)), we in depth compared results, discussion and conclusions of all of the selected papers with our directional sub-hypotheses (H0.1, H0.2, H0.3, H0.4 and H0.5) (in line with [53,57,71]).

Finally, in order to analyze the reasons for any disagreements among the studies about the direction of EP-BP relationship (see Table 1, sub-question (iv)), we discussed the not concordant results concerning the direction of EP-BP in the selected papers focusing on the main relevant factors that may have influenced the conclusions of these studies. 
Table 1. Synthesis of the research steps, tools and methods.

\begin{tabular}{|c|c|c|c|c|c|c|}
\hline & Sub-Question of Research & & Research Steps & Database Adopted in the Step & Type of Analysis & Research Keys \\
\hline \multirow[t]{2}{*}{ (i) } & \multirow[t]{2}{*}{$\begin{array}{l}\text { Which recent papers address the } \\
\text { relationship between EP and BP } \\
\text { of enterprises? }\end{array}$} & $\begin{array}{l}\text { Step } \\
\text { (I) }\end{array}$ & $\begin{array}{l}\text { Selection of papers related to EP } \\
\text { and BP of enterprises } \\
\begin{array}{ll}\text { (I-A) } & \text { Data Screening } \\
\text { (I-B) } & \text { Data Cleaning }\end{array}\end{array}$ & $\begin{array}{l}\text { (I-A) Selection with International } \\
\text { Database ISI Web of Knowledge } \\
\text { and considering specific editors' } \\
\text { libraries, as Emerald Insight, } \\
\text { Science Direct, Springer Library, } \\
\text { Wiley Library } \\
\text { (I-B) Selection of the papers searched } \\
\text { in Step (I-A) }\end{array}$ & $\begin{array}{l}\text { Survey of papers published in scientific } \\
\text { journals from } 2000 \text { to } 2015\end{array}$ & $\begin{array}{l}\text { (I-A) Search of terms: "environmental } \\
\text { performance" or "sustainable } \\
\text { performance" or "ecological } \\
\text { performance", and "business } \\
\text { performance" or "economic } \\
\text { performance" or "financial } \\
\text { performance", and "relationship", } \\
\text { and "firm" or "company" or } \\
\text { "enterprise", in: title, abstract, } \\
\text { keywords and/ or text. } \\
\text { (I-B) } \\
\text { Analysis of the contents in the full } \\
\text { text of each paper searched in } \\
\text { (I-A), and evaluation of their } \\
\text { coherency with the research } \\
\text { sub-question (i) }\end{array}$ \\
\hline & & $\begin{array}{l}\text { Step } \\
\text { (II) }\end{array}$ & $\begin{array}{l}\text { Analysis of the papers related to } \\
\text { EP-BP relationship }\end{array}$ & All papers selected in Step (I-B) & $\begin{array}{l}\text { Analysis of the text to consider type and } \\
\text { contents of each research effort }\end{array}$ & $\begin{array}{l}\text { Comparison of papers in terms of: } \\
\text { country of research, industrial sector } \\
\text { considered, type of research conducted, } \\
\text { sample of companies investigated, } \\
\text { research goals, environmental and } \\
\text { business variables considered to assess } \\
\text { firms' performances, } \\
\text { research conclusions }\end{array}$ \\
\hline (ii) & $\begin{array}{l}\text { Is there a consensus among the } \\
\text { authors to declare the existence of } \\
\text { EP-BP relationship? }\end{array}$ & $\begin{array}{l}\text { Step } \\
\text { (III) }\end{array}$ & $\begin{array}{l}\text { Verify if, within the selected } \\
\text { papers, an EP-BP } \\
\text { relationship exists }\end{array}$ & All papers selected in Step (II) & $\begin{array}{l}\text { Analysis of all text to consider results, } \\
\text { discussion and conclusions }\end{array}$ & $\begin{array}{l}\text { Confirmation or denial of the research } \\
\left.\text { hypotheses: } \mathrm{H} 0 \text { and } \mathrm{H} 1{ }^{*}\right)\end{array}$ \\
\hline (iii) & $\begin{array}{l}\text { Is there a specific direction of the } \\
\text { EP-BP relationship? }\end{array}$ & $\begin{array}{l}\text { Step } \\
\text { (IV) }\end{array}$ & $\begin{array}{l}\text { Verifying what type of EP-BP } \\
\text { relationship there is in } \\
\text { selected papers }\end{array}$ & $\begin{array}{l}\text { All papers in Step (III) that confirm H0 } \\
\text { hypothesis }\left(^{*}\right)\end{array}$ & $\begin{array}{l}\text { Analysis of all text to consider results, } \\
\text { discussion and conclusions }\end{array}$ & $\begin{array}{l}\text { Confirmation or denial of the research } \\
\text { sub-hypotheses: H0.1, H0.2, H0.3, H0.4, } \\
\text { and H0.x }\left(^{* *}\right)\end{array}$ \\
\hline (iv) & $\begin{array}{l}\text { What are the reasons that do not } \\
\text { lead the studies to a consensus } \\
\text { view in the direction of } \\
\text { EP-BP relationship? }\end{array}$ & $\begin{array}{l}\text { Step } \\
\text { (V) }\end{array}$ & $\begin{array}{l}\text { Analyzing the reasons that lead to } \\
\text { different conclusions in } \\
\text { selected papers }\end{array}$ & $\begin{array}{l}\text { All papers in Step (III) that confirm H0 } \\
\text { hypothesis }\left(^{*}\right)\end{array}$ & $\begin{array}{l}\text { Discussion about the results of } \\
\text { Steps (III) and (IV) }\end{array}$ & $\begin{array}{l}\text { Research objectives and goals, } \\
\text { research methodology, environmental } \\
\text { and business variables considered to } \\
\text { assess firms' performances, } \\
\text { research conclusions }\end{array}$ \\
\hline
\end{tabular}

${ }^{(*)}$ Research hypotheses: H0: There is a relationship between EP and BP; H1: There is no relationship between EP and BP; $\left(^{* *}\right)$ Research sub-hypotheses: H0.1: Better EP determines inverse, non-linear, U-shaped, and conditional) 


\section{Results}

\subsection{Selection of Papers Related to the EP-BP Relationship of Enterprises (Step I)}

The first step of this research (conducted as specified in Table 1) was to identify the papers published from 2000 to 2015 that contained the terms "environmental performance", "economic performance", "enterprise" and "relationship" (or synonyms) in their titles, abstracts, keywords or texts.

Two hundred twenty-nine papers were initially selected in Step (I-A) Data Screening: this first selection represents all the papers published in ISI journals from 2000 to 2015 that include in their title, abstract, keywords, or full text our research keywords. In Step (I-B) Data Cleaning, deeper examination of the contents of these papers led us to exclude 182 papers because although they discussed EP and/or BP, they did not specifically explore the EP-BP relationship at the firm level. Specifically, the papers excluded from the research sample dealt with the following subjects: 22 papers analyzed the relationship between different environmental aspects, 14 papers discussed the business conditions and motivations surrounding the adoption of environmental practices, 30 papers proposed methods to measure EP and BP in a combined way, 27 papers discussed the relationship between environmental practices or strategies and business returns, 16 papers considered the relationship between environmental innovation or eco-design and BP, nine papers analyzed the relationship between EP and $\mathrm{BP}$ at the regional or national level (not at the company level), 40 papers considered the relationship between corporate social responsibility and BP, and, finally, 24 papers discussed specific environmental practices that were not related to BP.

Therefore, we finally selected 47 papers published in 18 scientific journals from 2000 to 2015: these papers explored a deep relationship between the EP and the BP at the firm level. Figure 1 shows the trend of these papers between 2000 and 2015: the discussion about EP-BP relationship at the firms' level started from 2005, and most of the papers concerning this debate were published in the last years (45\% from 2013 to 2015). We can also highlight that the debate regarding the EP-BP relationship is currently presented in several journals dealing with various disciplines, such as environment, management, business and ethics.

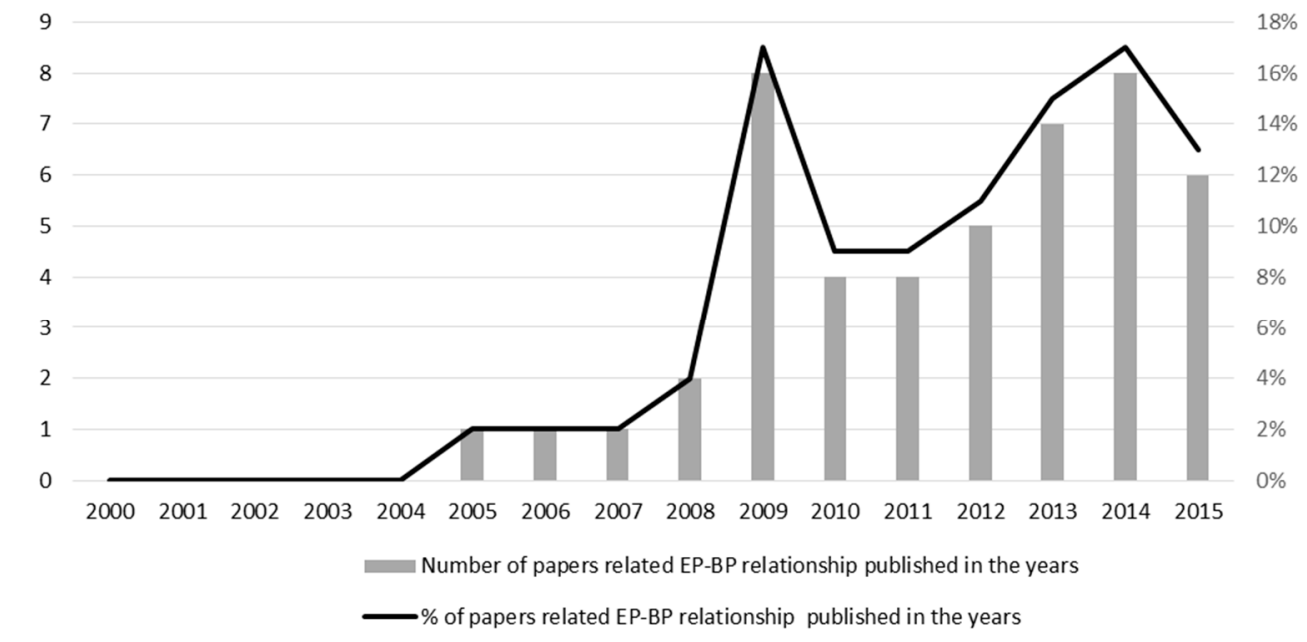

Figure 1. Trend of the papers published from 2000 to 2015 concerning the EP-BP relationship at the firms' level.

\subsection{Analysis of the Papers Related to the Relationship between EP and BP of Enterprises (Step II)}

The second research step (as specified in Table 1) called for comparing the 47 papers selected in terms of: the type of research conducted, research goals, variables considered to measure EP and BP and research conclusions. A summary of the results is given in Tables 2 and 3. 
Table 2. Summary of the papers concerning the EP-BP relationship of enterprises.

\begin{tabular}{|c|c|c|c|c|}
\hline Paper & Country & Industrial Sector & Research Type * & Sample of Companies \\
\hline Wagner [25] & Germany, Italy, the Netherlands and UK & Paper industry & Survey using data from public database & 37 firms \\
\hline Earnhart and Lizal [72] & Czech Republic & Not specified & Survey using data from public database & 2628 observations during 6 years \\
\hline Kranjc et al. [73] & Slovenia & Food process & Multiple case study & $\begin{array}{l}1 \text { beet sugar plant with } 2 \text { alternative } \\
\text { optimization process designs }\end{array}$ \\
\hline Galdeano-Gómez [74] & Spain & Fresh fruit and vegetables & Survey using data from public database & $\begin{array}{l}56 \text { farming-marketing firms located in } \\
\text { Andalusia (South of Spain) }\end{array}$ \\
\hline Yamaguchi and van Kooten [75] & United States and Canada & Forest products industry & Survey using data from public database & 21 companies \\
\hline Boons and Wagner [37] & Not applicable & Not applicable & Literature review & Not specified \\
\hline Iraldo et al. [76] & European Union & Not specified & Survey using data from public database & $\begin{array}{l}70 \text { private organizations EMAS registered and } \\
31 \text { not EMAS registered }\end{array}$ \\
\hline López-Gamero et al. [77] & Spain & $\begin{array}{l}\text { Hotels and firms affected by the } \\
\text { IPPC law }\end{array}$ & Survey with interviews & $\begin{array}{l}3900 \text { hotels and } 4187 \text { firms affected by the IPPC } \\
\text { law involved, } 240 \text { hotels and } 208 \text { firms respondent }\end{array}$ \\
\hline Mazzanti and Zoboli [78] & Italy & Various sectors & Survey using data from public database & 319 firms in 29 sector branches \\
\hline Menguc et al. [79] & New Zealand & Various sectors & Survey with interview & 325 firms involved, 150 respondent \\
\hline Molina-Azorín et al. [80] & Not applicable & Not applicable & Literature review & 32 papers until 2008 \\
\hline Molina-Azorín et al. [50] & Spain & Hotel industry & Survey with interviews & 3900 hotels involved , 301 respondent \\
\hline Yu et al. [81] & European Union & Various sectors & $\begin{array}{l}\text { Survey using data from a previous } \\
\text { European project }\end{array}$ & 51 companies \\
\hline Horváthová [51] & Not applicable & Not applicable & Literature review & 37 empirical studies \\
\hline López-Gamero et al. [82] & Spain & $\begin{array}{l}\text { Various sectors affected by } \\
\text { IPPC law }\end{array}$ & Survey using data from public database & 208 firms \\
\hline Wagner [83] & United States & Various sectors & Survey using data from public database & 358 firms \\
\hline Zeng et al. [84] & China & Manufacturing industry & Survey with interviews & 500 companies involved, 125 respondent \\
\hline Heras-Saizarbotoria et al. [85] & Basque region (Spain) & Various sectors & Survey using data from public database & All companies in Basque region \\
\hline Iwata and Okada [86] & Japan & $\begin{array}{l}\text { Various sectors with clean and } \\
\text { dirty processes }\end{array}$ & Survey using data from public database & 268 manufacturing firms \\
\hline Salama et al. [87] & United Kingdom & Various sectors & Survey using data from public database & 567 firms \\
\hline Zeng et al. [88] & China & Various sectors & Survey with interviews & 500 SMEs involved , 137 respondent \\
\hline Figge and Hahn [70] & Worldwide & Automotive & Survey using data from public database & 16 car makers worldwide \\
\hline Horváthová [89] & Czech Republic & Various sectors & Survey using data from public database & 136 firms \\
\hline Lioui and Sharma [90] & Worldwide & Various sectors & Survey using data from public database & Approximately 3100 firms \\
\hline Perez-Calderon et al. [91] & Worldwide & Various sectors except financial & Survey using data from public database & 122 firms \\
\hline
\end{tabular}


Table 2. Cont.

\begin{tabular}{|c|c|c|c|c|}
\hline Paper & Country & Industrial Sector & Research Type * & Sample of Companies \\
\hline Tang et al. [92] & United States & Various sectors & Survey using data from public database & 500 largest US enterprises \\
\hline Albertini [54] & Not applicable & Not applicable & Literature review & 52 studies \\
\hline Apeaning and Thollander [93] & Ghana & Various sectors & Survey with interviews & 76 companies involved, 34 respondent \\
\hline Dixon-Fowler et al. [52] & Not applicable & Not applicable & Literature review & 39 studies \\
\hline Dragomir [94] & European Union & Various sectors & Survey using data from public database & 77 large companies \\
\hline Fujii et al. [63] & Japan & Various manufacturing sectors & Survey using data from public database & 758 firms \\
\hline Youn et al. [95] & Korea & Various sectors & Survey with interviews & 400 companies involved, 142 respondent \\
\hline De Burgos-Jiménez et al. [96] & Wales (United Kingdom) & Various sectors & $\begin{array}{l}\text { Survey with interviews and using data } \\
\text { from public database }\end{array}$ & 2122 companies \\
\hline Endrikat et al. [56] & Not applicable & Not applicable & Literature review & 149 studies \\
\hline Gallego-Álvarez et al. [97] & Worldwide & Various sectors & Survey using data from public database & 855 multinational companies \\
\hline Gotschol et al. [64] & Italy & Various sectors & Survey with interviews & Sample random selected , 240 responden \\
\hline Guenther and Hoppe [53] & Not applicable & Not applicable & Literature review & Not specified \\
\hline Moon et al. [98] & United States & Various industrial sectors & Survey using data from public database & 393 large US firms \\
\hline Ortas et al. [99] & Worldwide & Various sectors & Survey using data from public database & 3900 companies \\
\hline Qi et al. [100] & China & Various industrial sectors & Survey using data from public database & 39 industries \\
\hline Wang et al. [101] & Australia & Various industrial sectors & Survey using data from public database & 69 firms \\
\hline Larrán Jorge et al. [102] & Spain & Various sectors & Survey with interviews & 4860 SMEs involved, 481 respondent \\
\hline Misani and Pogutz [103] & Worldwide & Carbon intensive industries & Survey using data from public database & 127 global firms \\
\hline Muhammad et al. [104] & Australia & Various sectors & Survey using data from public database & 76 companies \\
\hline Muhammad et al. [105] & Australia & Various sectors & Survey using data from public database & 76 companies \\
\hline Sánchez-Medina et al. [106] & Mexico & Pottery handicraft sector & $\begin{array}{l}\text { Survey with interview and using data } \\
\text { from public database }\end{array}$ & 200 firms involved, 186 respondent \\
\hline Trumpp and Guenther [71] & Worldwide & Various services sectors & Survey using data from public database & 2361 firm-during 5 years \\
\hline
\end{tabular}


Table 3. Main contents of the papers concerning the EP-BP relationship of enterprises.

\begin{tabular}{|c|c|c|c|}
\hline Paper & Environmental Performance/Variables Considered & Business Performance/Variables Considered & Conclusive Propositions \\
\hline \multirow{2}{*}{ Wagner [25] } & \multirow{2}{*}{$\begin{array}{l}\text { Emission of } \mathrm{COD}, \mathrm{SO}_{4}, \mathrm{NO}_{x} \text {, Energy input, Water input (called } \\
\text { "environmental performance") }\end{array}$} & \multirow{2}{*}{$\begin{array}{l}\text { Return on capital employed, Return on equity, Return on } \\
\text { sales (called "business performance") }\end{array}$} & $\begin{array}{l}\text { A predominantly negative relationship between environmental and } \\
\text { economic performance is found for the emissions-based index. Whereas for the inputs-based } \\
\text { index no significant link is found. }\end{array}$ \\
\hline & & & $\begin{array}{l}\text { The EP-BP relationship is more positive for firms with pollution } \\
\text { prevention-oriented strategies. }\end{array}$ \\
\hline \multirow{2}{*}{$\begin{array}{l}\text { Earnhart and } \\
\text { Lizal [72] }\end{array}$} & \multirow{2}{*}{$\begin{array}{l}\left.\text { Air pollutants ( } \mathrm{CO}^{\mathrm{SO}} \mathrm{O}_{2}, \mathrm{NO}_{x}, \mathrm{PM}\right) \\
\text { (called "environmental performance") }\end{array}$} & \multirow{2}{*}{$\begin{array}{l}\text { Production, Lagged profits, State ownership, Investment } \\
\text { found ownership, Bank ownershiph, Portfolio company } \\
\text { owner, Citizen ownership, Strategic investor ownership, } \\
\text { Foreign investor ownership, Concentration, Invers Mills } \\
\text { ratio (called "financial performance") }\end{array}$} & $\begin{array}{l}\text { Since liquidity constraints limit investment in pollution reduction, subsidizing investments in } \\
\text { environmental improvement technologies is beneficial. }\end{array}$ \\
\hline & & & $\begin{array}{l}\text { The results highlight the importance of using multiple measures of environmental and } \\
\text { financial performance. }\end{array}$ \\
\hline \multirow{2}{*}{ Kranjc et al. [73] } & \multirow{2}{*}{$\begin{array}{l}\text { Climate change, Acidification, Eutrophication, Photochemical ozone } \\
\text { creation, Human toxicity (called "environmental impacts") }\end{array}$} & \multirow{2}{*}{$\begin{array}{l}\text { Monetary values to environmental impacts, } \\
\text { Raw material price, Energy price, Transport price } \\
\text { (called "economic performances") }\end{array}$} & $\begin{array}{l}\text { The same design plant option is preferable from the economic and } \\
\text { environmental perspective. }\end{array}$ \\
\hline & & & Energy minimization is one of the most important issues within the context of sustainability. \\
\hline $\begin{array}{l}\text { Galdeano } \\
\text {-Gómez [74] }\end{array}$ & $\begin{array}{l}\text { Annual expenditure on environmental practices over sales } \\
\text { (called "environmental performance") }\end{array}$ & $\begin{array}{l}\text { Sales margins, Market share } \\
\text { (called "economic performance") }\end{array}$ & $\begin{array}{l}\text { The environmental differentiation show a positive impact on profitability and market share } \\
\text { and it implies greater environmental performance. }\end{array}$ \\
\hline \multirow{2}{*}{$\begin{array}{l}\text { Yamaguchi and } \\
\text { van Kooten [75] }\end{array}$} & \multirow{2}{*}{$\begin{array}{l}\text { Chemical pollutant }\left(\mathrm{as}_{2} \mathrm{CO}_{2} \text { and } \mathrm{CH}_{3} \mathrm{OH} \text { ) }\right. \\
\text { (called "pollution intensity") }\end{array}$} & \multirow{2}{*}{$\begin{array}{l}\text { Return on capital employed } \\
\text { (called "corporate financial performance") }\end{array}$} & $\begin{array}{l}\text { More successful firms, measured by profitability, innovate and invest in cleaner and more } \\
\text { cost-efficient technologies. }\end{array}$ \\
\hline & & & $\begin{array}{l}\text { We cannot confirm that reductions in pollution intensity are associated with improvements in } \\
\text { economic performance. }\end{array}$ \\
\hline $\begin{array}{l}\text { Boons and } \\
\text { Wagner [37] }\end{array}$ & $\begin{array}{l}\text { Environmental impacts of production and consumption (called } \\
\text { "production and consumption system") }\end{array}$ & $\begin{array}{l}\text { Costs and benefits at the individual firms, Proactive } \\
\text { strategies and competitive advantage at the market level, } \\
\text { Economic growth at the economic level } \\
\text { (called "system levels") }\end{array}$ & $\begin{array}{l}\text { The mixed results obtained from the literature review can be better understood if you analyze } \\
\text { them in relation to different system boundaries: at the firm level, at the market level, at the } \\
\text { economic level. }\end{array}$ \\
\hline Iraldo et al. [76] & $\begin{array}{l}\text { EMAS maturity, Environmental targets, Environmental } \\
\text { improvement, Suppliers' environmental measures (called } \\
\text { "characteristics of EMS") }\end{array}$ & $\begin{array}{l}\text { Market performance, Innovation capability, } \\
\text { Resource efficiency, Intangible assets (called } \\
\text { "variables of competitiveness") }\end{array}$ & $\begin{array}{l}\text { Environmental performance has a positive impact on innovations, but effects on other } \\
\text { competitive variables are not strongly supported. }\end{array}$ \\
\hline $\begin{array}{l}\text { López-Gamero } \\
\text { et al. [77] }\end{array}$ & $\begin{array}{l}\text { Efficient use resource, Reduction of emissions, } \\
\text { Reduction of residues, Reduction of acoustic pollution } \\
\text { (called "environmental performance") }\end{array}$ & $\begin{array}{l}\text { New firm resources, Competitive advantage, } \\
\text { Financial performance as subjective perception (called } \\
\text { "economic performance") }\end{array}$ & $\begin{array}{l}\text { Firm's resources and competitive advantage act as mediator variables for a positive } \\
\text { relationship between environmental protection and financial performance. }\end{array}$ \\
\hline \multirow{2}{*}{$\begin{array}{l}\text { Mazzanti and } \\
\text { Zoboli }[78]\end{array}$} & \multirow{2}{*}{$\begin{array}{l}\text { Emission intensity of value added (in terms of } \mathrm{CO}_{2}, \mathrm{CH}_{4}, \mathrm{NO}_{x}, \mathrm{SO}_{x} \text {, } \\
\text { NMVOC, } \mathrm{PM}_{10} \text { emissions) (called "environmental efficiency") }\end{array}$} & \multirow{2}{*}{ Value added per employee (called "productivity measure") } & $\begin{array}{l}\text { For most air emission categories there is a positive relationship between labor productivity } \\
\text { and environmental efficiency. }\end{array}$ \\
\hline & & & $\begin{array}{l}\text { Services show a complementary relationship, while industry associates better labor } \\
\text { productivity with lower GHG. }\end{array}$ \\
\hline \multirow{3}{*}{ Menguc et al. [79] } & \multirow{3}{*}{$\begin{array}{l}\text { Pollution prevention, Top management support, Customers' } \\
\text { environmental sensitivity, Government regulations, Environmental } \\
\text { dynamism (called "proactive environmental strategy") }\end{array}$} & \multirow{3}{*}{$\begin{array}{l}\text { Sales growth, Profit growth, Entrepreneurial orientation } \\
\text { (called "firm's performance") }\end{array}$} & Proactive environmental strategy can benefit sales and profit growth. \\
\hline & & & Government regulation increases the positive relationship between EP and BP. \\
\hline & & & Consumer sensitivity had a direct effect in this relationship. \\
\hline \multirow{2}{*}{$\begin{array}{l}\text { Molina-Azorín } \\
\text { et al. [80] }\end{array}$} & \multirow{2}{*}{$\begin{array}{l}\text { Environmental management variables (as technical and } \\
\text { organizational activities), Environmental performance variables (as } \\
\text { environmental impacts and effects in the natural environmental) } \\
\text { (called "environmental variables") }\end{array}$} & \multirow[t]{2}{*}{ Financial performance (called "financial variables") } & Results derived by many studies are mixed. \\
\hline & & & Studies with a positive impact of environment on financial performance are predominant. \\
\hline
\end{tabular}


Table 3. Cont

\begin{tabular}{|c|c|c|c|}
\hline Paper & Environmental Performance/Variables Considered & Business Performance/Variables Considered & Conclusive Propositions \\
\hline $\begin{array}{l}\text { Molina-Azorín } \\
\text { et al. [50] }\end{array}$ & $\begin{array}{l}\text { Technical practices (such as energy and water saving activities), } \\
\text { Organizational practices (such as quantification of environmental } \\
\text { costs and savings, personal training in environmental issues) (called } \\
\text { "environmental practices") }\end{array}$ & $\begin{array}{l}\text { Occupancy rate per room, Gross operative profit, Gross } \\
\text { operative profit per available room per day } \\
\text { (called "firm's performance") }\end{array}$ & Environmental practices impact significantly on several performance variables. \\
\hline Yu et al. [81] & $\begin{array}{l}\text { Air pollutants emission (such as } \mathrm{CO}_{2}, \mathrm{NO}_{x}, \mathrm{SO}_{x}, \mathrm{VOC} \text {, and } \mathrm{CH}_{4} \text { ), } \\
\text { Waste generation, Water use (called "environmental resources") }\end{array}$ & $\begin{array}{l}\text { Sustainable value margin, Return to cost ratio (called } \\
\text { "sustainable value") }\end{array}$ & $\begin{array}{l}\text { Results do not find a positive relationship between EP and BP both for all the companies' } \\
\text { sample both for the carbon-intensive sector. }\end{array}$ \\
\hline \multirow{3}{*}{ Horváthová [51] } & \multirow{3}{*}{ All the environmental variables adopted in the papers analyzed } & \multirow{3}{*}{ Not specified } & The portfolio studies tend to report a negative link between EP and BP. \\
\hline & & & $\begin{array}{l}\text { The positive link is found more frequently in common law countries than in } \\
\text { civil law countries. }\end{array}$ \\
\hline & & & $\begin{array}{l}\text { It is fundamental the adoption of an appropriate time of coverage to establish a positive link } \\
\text { between EP and BP. }\end{array}$ \\
\hline $\begin{array}{l}\text { López-Gamero } \\
\text { et al. [82] }\end{array}$ & $\begin{array}{l}\text { Operational aspects of environmental management, } \\
\text { Technical aspects of environmental management (called } \\
\text { "environmental management") }\end{array}$ & $\begin{array}{l}\text { Competitive advantage on costs, Competitive advantage on } \\
\text { differentiation, Growth in added value, Economic } \\
\text { development, Financial profitability (called "competitive } \\
\text { advantage and financial performance") }\end{array}$ & $\begin{array}{l}\text { Environmental command-and-control legislation is a not significant influence on } \\
\text { environmental management and managerial perception. Investment in proactive } \\
\text { environmental management contributes to increase competitiveness. }\end{array}$ \\
\hline Wagner [83] & $\begin{array}{l}\text { Rating of corporate social responsibility and environmental } \\
\text { management (called "environmental and social performance") }\end{array}$ & Tobin's q (called "economic performance") & $\begin{array}{l}\text { The EP has a direct effect in the BP, and the corporate social responsibility } \\
\text { has only moderate effect. }\end{array}$ \\
\hline \multirow[b]{2}{*}{ Zeng et al. [84] } & \multirow{2}{*}{$\begin{array}{l}\text { Employee environmental consciousness, Waste reduction, Rules for } \\
\text { cleaner production, Cleaner production policy, Recyclability, } \\
\text { Packaging reducing, Energy efficiency, Clean technologies, } \\
\text { Renewable resources use, durability of product, investment in } \\
\text { environmental protection, technology innovation (called "indexes of } \\
\text { cleaner production") }\end{array}$} & \multirow{2}{*}{$\begin{array}{l}\text { Profitability, Increased rate of net profit, Return on equity, } \\
\text { Market share, Corporate reputation, Shareholders' } \\
\text { confidence (called "business performance") }\end{array}$} & Results find an overall positive impact of cleaner production on firms' business performance. \\
\hline & & & $\begin{array}{l}\text { Low-cost cleaner production variables have a larger contribution to financial performance; } \\
\text { high-cost cleaner production variables have a larger contribution to } \\
\text { non-financial performance. }\end{array}$ \\
\hline $\begin{array}{l}\text { Heras-Saizarbotoria } \\
\text { et al. [85] }\end{array}$ & $\begin{array}{l}\text { ISO } 14001 \text { certification, ISO } 14001 \text { certification data (called } \\
\text { "environmental performance") }\end{array}$ & Return on assets (called "financial performance") & $\begin{array}{l}\text { Firms with better than average performance have a greater propensity to pursue } \\
\text { accreditation but there is no evidence that improvements in performance follow certification. }\end{array}$ \\
\hline \multirow{2}{*}{$\begin{array}{l}\text { Iwata and } \\
\text { Okada [86] }\end{array}$} & \multirow{2}{*}{$\begin{array}{l}\text { Greenhouse gas emissions, Waste } \\
\text { (called "environmental performance") }\end{array}$} & \multirow{2}{*}{$\begin{array}{l}\text { Return on equity, Return on assets, Return on investment, } \\
\text { Return on invested capital, Return on sales, Tobin's q (called } \\
\text { "financial performance") }\end{array}$} & $\begin{array}{l}\text { Reduction of waste production does not generally have significant effects } \\
\text { on financial performance. }\end{array}$ \\
\hline & & & $\begin{array}{l}\text { Greenhouse gas reduction lead to increase in financial performance in the clean industries, } \\
\text { but it does not have significant effects on financial performance in dirty industries. }\end{array}$ \\
\hline \multirow[b]{2}{*}{ Salama et al. [87] } & \multirow{2}{*}{$\begin{array}{l}\text { Investment in Community and environmental responsibility (called } \\
\text { "environmental performance") }\end{array}$} & \multirow[b]{2}{*}{ Market risk (called "firm risk") } & A company's environmental performance is inversely related to its systematic financial risk. \\
\hline & & & $\begin{array}{l}\text { Market incentives for investment in environmental practices may complement } \\
\text { regulatory alternatives. }\end{array}$ \\
\hline Zeng et al. [88] & $\begin{array}{l}\text { Contamination release, energy consumption, suppliers with } \\
\text { environmental performance, environmental management system, } \\
\text { cleaner production activities, staff training, environmental auditing } \\
\text { (called "environmental performance") }\end{array}$ & $\begin{array}{l}\text { Sales, Profitability, Inventory turnover, Return on equity, } \\
\text { Market share, Sales region, Number of customers (called } \\
\text { "economic performance") }\end{array}$ & $\begin{array}{l}\text { Environmental performance is moderately correlated with financial indices, but not } \\
\text { significantly with non-financial indices. }\end{array}$ \\
\hline $\begin{array}{l}\text { Figge and } \\
\text { Hahn [70] }\end{array}$ & Environmental value creation (called "environmental performance") & Economic value creation (called "economic performance") & $\begin{array}{l}\text { Using the opportunity cost based approach, companies adopt strategies that create economic } \\
\text { and environmental value and maximize both environmental and economic performance. }\end{array}$ \\
\hline Horváthová [89] & Amount of emissions (called "environmental performance") & $\begin{array}{l}\text { Return on assets, Return on equity (called "financial } \\
\text { performance") }\end{array}$ & $\begin{array}{l}\text { There is not sufficient evidence about the intertemporal effect of EP on BP. The time-period is } \\
\text { significant in the EP-BP relationship. }\end{array}$ \\
\hline $\begin{array}{l}\text { Lioui and } \\
\text { Sharma [90] }\end{array}$ & $\begin{array}{l}\text { Firms' environmental corporate social responsibility rating (called } \\
\text { "environmental corporate social responsibility") }\end{array}$ & $\begin{array}{l}\text { Return on assets, R \& D, Tobin's q (called "financial } \\
\text { performance") }\end{array}$ & $\begin{array}{l}\text { The relationship between EP and direct BP (such as financial performance ROA and Tobin's } \mathrm{s} \text { ) } \\
\text { is negative. However, the relationship between EP and indirect } \mathrm{BP} \text { (such as } \mathrm{R} \& \mathrm{D} \text { ) is positive. }\end{array}$ \\
\hline \multirow{2}{*}{$\begin{array}{l}\text { Perez-Calderon } \\
\text { et al. [91] }\end{array}$} & \multirow{2}{*}{$\begin{array}{l}\text { Water consumption, Energy consumption, emissions to air (called } \\
\text { "environmental performance") }\end{array}$} & \multirow{2}{*}{$\begin{array}{l}\text { Return on assets, Return on sales, Market-to-book ratio } \\
\text { (called "economic and financial performance") }\end{array}$} & $\begin{array}{l}\text { The companies, which show greatest efficiency in energy and water consumption, are also } \\
\text { the ones, which achieved the best economic and financial profitability ratios. }\end{array}$ \\
\hline & & & $\begin{array}{l}\text { This relationship is not demonstrated in the case of non-environmentally sensitive companies } \\
\text { and in the case of stock market index. }\end{array}$ \\
\hline
\end{tabular}


Table 3. Cont.

\begin{tabular}{|c|c|c|c|}
\hline Paper & Environmental Performance/Variables Considered & Business Performance/Variables Considered & Conclusive Propositions \\
\hline \multirow{2}{*}{ Tang et al. [92] } & \multirow{2}{*}{$\begin{array}{l}\text { Climate change, Pollution, Product impact, Environmental } \\
\text { stewardship, Environmental management } \\
\text { (called "environmental governance") }\end{array}$} & \multirow{2}{*}{ Tobin's q (called "economic performance") } & EP enhances green reputation, which improves corporate reputation and hence EP. \\
\hline & & & EP is positively associated with customer satisfaction, which in turn improves EP. \\
\hline Albertini [54] & All the environmental variables adopted in the papers analyzed & All the financial variables adopted in the papers analyzed & $\begin{array}{l}\text { It is possible to confirm the positive relationship between EP and BP. } \\
\text { The relationship is significantly influenced by EP and BP measures, regional differences, } \\
\text { activity sector, time-period. }\end{array}$ \\
\hline $\begin{array}{l}\text { Apeaning and } \\
\text { Thollander [93] }\end{array}$ & $\begin{array}{l}\text { Energy efficiency measures, Energy efficient technologies (called } \\
\text { "energy efficiency") }\end{array}$ & $\begin{array}{l}\text { Access to capital, Hidden costs, Business risk, Imperfect } \\
\text { information, Split incentives, Market related, Organizational } \\
\text { and behavioral factors (called "barriers and driving forces to } \\
\text { energy efficiency") }\end{array}$ & $\begin{array}{l}\text { The most important factors inhibiting the energy efficiency are "luck of budget funding" and } \\
\text { "access to capital". }\end{array}$ \\
\hline \multirow{3}{*}{$\begin{array}{l}\text { Dixon-Fowler } \\
\text { et al. [52] }\end{array}$} & \multirow{3}{*}{ Environmental strategy (called "environmental moderator") } & \multirow{3}{*}{ Firm characteristics (called "economic moderators") } & The positive relationship EP-BP exist. \\
\hline & & & $\begin{array}{l}\text { Moderator variables are: USA vs. international market, market-measures of BP, institutional } \\
\text { vs. self-reported data. }\end{array}$ \\
\hline & & & $\begin{array}{l}\text { Moderator variables are not: small vs. large firms, proactive vs. end-of-pipe environmental } \\
\text { strategies, different EP measures. }\end{array}$ \\
\hline \multirow[b]{2}{*}{ Dragomir [94] } & \multirow[b]{2}{*}{ Greenhouse gas emissions (called "environmental performance") } & \multirow[b]{2}{*}{ Return on assets (called "firm performance") } & There is no definitive conclusion on the EP-BP relationship. \\
\hline & & & $\begin{array}{l}\text { Higher costs, but also higher revenues determine a neutral relationship between corporate } \\
\text { social responsibility activities and firm financial performance. }\end{array}$ \\
\hline \multirow{2}{*}{ Fujii et al. [63] } & \multirow{2}{*}{$\begin{array}{l}\mathrm{CO}_{4} \text { emissions, Toxic chemical substances emissions (called } \\
\text { "environmental performance") }\end{array}$} & \multirow{2}{*}{$\begin{array}{l}\text { Return on assets, Return on sales, Capital turnover } \\
\text { (called "economic performance") }\end{array}$} & $\begin{array}{l}\text { There is a significant positive relationship between } \mathrm{CO}_{2} \text { emissions reduction and } \\
\text { financial performance. }\end{array}$ \\
\hline & & & $\begin{array}{l}\text { There is a significant inverted U-shaped relationship between return on assets and } \\
\text { environmental performance. }\end{array}$ \\
\hline \multirow{2}{*}{ Youn et al. [95] } & \multirow{2}{*}{$\begin{array}{l}\text { Waste reduction, Recycling, Reuse, Material substitution, Internal } \\
\text { environmental management, Eco-design, Energy consumption } \\
\text { (called "environmental performance") }\end{array}$} & \multirow{2}{*}{$\begin{array}{l}\text { Organizational reputation, Market penetration, Profitability, } \\
\text { Customer satisfaction and loyalty } \\
\text { (called "business performance") }\end{array}$} & $\begin{array}{l}\text { Strategic supply chain partnership facilitates the implementation of environmental supply } \\
\text { chain management. }\end{array}$ \\
\hline & & & $\begin{array}{l}\text { The improved environmental performance determines the improvement } \\
\text { of business performance. }\end{array}$ \\
\hline \multirow{2}{*}{$\begin{array}{l}\text { De Burgos-Jiménez } \\
\text { et al. [96] }\end{array}$} & \multirow[t]{2}{*}{ Waste production (called "environmental performance") } & \multirow{2}{*}{$\begin{array}{l}\text { Return on assets, Return on sales, Sales variation (called } \\
\text { "financial performance") }\end{array}$} & $\begin{array}{l}\text { The relationship between environmental strategy and financial performance is significant } \\
\text { and positive. }\end{array}$ \\
\hline & & & The EP-BP relationship is significant and positive. \\
\hline \multirow{2}{*}{$\begin{array}{l}\text { Endrikat } \\
\text { et al. [56] }\end{array}$} & \multirow{2}{*}{ All the environmental variables adopted in the papers analyzed } & \multirow{2}{*}{ All the financial variables adopted in the papers analyzed } & $\begin{array}{l}\text { There is a positive and partially bidirectional relationship between corporate environmental } \\
\text { performance and corporate financial performance. }\end{array}$ \\
\hline & & & $\begin{array}{l}\text { This relationship is stronger when the environmental strategy of firms is proactive rather } \\
\text { than reactive. }\end{array}$ \\
\hline $\begin{array}{l}\text { Gallego-Álvarez } \\
\text { et al. [97] }\end{array}$ & $\begin{array}{l}\text { Greenhouse gas emissions by sales volume } \\
\text { (called "environmental performance") }\end{array}$ & Return on assets (called "economic performance") & $\begin{array}{l}\text { In time of economic crisis, the synergy between environmental and financial performance is } \\
\text { higher and the companies continue to invest in sustainable projects in order to enhance } \\
\text { relations with their customers and stakeholders which reinforces their economic activity. }\end{array}$ \\
\hline \multirow{2}{*}{$\begin{array}{l}\text { Gotschol } \\
\text { et al. [64] }\end{array}$} & \multirow{2}{*}{$\begin{array}{l}\text { Reduction of air emissions, Reduction of solid/liquid waste, } \\
\text { Reduction of the amount of energy used, Reduction of consumption } \\
\text { for hazardous/toxic materials, Decrease of frequency of } \\
\text { environmental accidents (called "environmental performance") }\end{array}$} & \multirow{2}{*}{$\begin{array}{l}\text { Profit, Market share, Cost savings } \\
\text { (called "economic performance") }\end{array}$} & Environmental performance has a positive impact on economic performance in the long run. \\
\hline & & & $\begin{array}{l}\text { Better economic performance has a positive effect on environmental performance also in the } \\
\text { short run. }\end{array}$ \\
\hline \multirow{2}{*}{$\begin{array}{l}\text { Guenther and } \\
\text { Hoppe [53] }\end{array}$} & \multirow{2}{*}{$\begin{array}{l}\text { Strategic corporate environmental performance, Operational } \\
\text { corporate environmental performance, Environmental reporting, } \\
\text { Rating and ranking, Questionnaire-based (perceived) corporate } \\
\text { environmental performance, Environment-related events (called } \\
\text { "environmental categories") }\end{array}$} & \multirow{2}{*}{$\begin{array}{l}\text { Stock market, Stock market and account, Accounting, } \\
\text { Questionnaire-based (perceived) corporate financial } \\
\text { performance (called "financial categories") }\end{array}$} & The relationship between $\mathrm{EP}$ and $\mathrm{BP}$ is very complex. \\
\hline & & & $\begin{array}{l}\text { Authors propose a theoretical model to support the analysis of the direction of } \\
\text { EP-BP relationship. }\end{array}$ \\
\hline
\end{tabular}


Table 3. Cont.

\begin{tabular}{|c|c|c|c|}
\hline Paper & Environmental Performance/Variables Considered & Business Performance/Variables Considered & Conclusive Propositions \\
\hline \multirow{3}{*}{ Moon et al. [98] } & \multirow{3}{*}{$\begin{array}{l}\text { Environmental corrective actions, Toxic chemical emission intensity, } \\
\text { Penalty index value (called "environmental performance") }\end{array}$} & \multirow{3}{*}{ Return on assets (called "economic performance") } & Voluntary environmental programs had a positive effect on firm's economic performance. \\
\hline & & & Pollution reduction and prevention helps promote the "green" image. \\
\hline & & & Firm's poor environmental track record had a negative effect in its economic performance. \\
\hline \multirow{2}{*}{ Ortas et al. [99] } & \multirow{2}{*}{$\begin{array}{l}\text { Sustainable supply chain management } \\
\text { (called "sustainable performance") }\end{array}$} & \multirow{2}{*}{$\begin{array}{l}\text { Margins/Performance, Profitability/Shareholder loyalty, } \\
\text { Revenue/Customer loyalty (called "financial performance") }\end{array}$} & $\begin{array}{l}\text { There is a significant, bidirectional causation between sustainable supply chain management } \\
\text { and companies' margins and revenue. }\end{array}$ \\
\hline & & & This remained true during the bull market but not during the financial crisis. \\
\hline \multirow{2}{*}{ Qi et al. [100] } & \multirow{2}{*}{$\begin{array}{l}\mathrm{SO}_{3} \text { emissions per unit of industry value added (called } \\
\text { "environmental performance") }\end{array}$} & \multirow{2}{*}{$\begin{array}{l}\text { Return on assets, Shipment value growth rate, Assets/debt } \\
\text { ratio (called "financial performance") }\end{array}$} & The EP-BP link is consistent with the industry-level analysis. \\
\hline & & & The EP-BP relationship is stronger when level of slack resources is high. \\
\hline Wang et al. [101] & Greenhouse gas emissions (called "environmental performance") & $\begin{array}{l}\text { Tobin's q, Share price, Share quantities (called "financial } \\
\text { performance") }\end{array}$ & $\begin{array}{l}\text { There is a positive correlation between greenhouse gas emissions reduction and corporate } \\
\text { financial performance in all industry sectors. }\end{array}$ \\
\hline $\begin{array}{l}\text { Larrán Jorge } \\
\text { et al. [102] }\end{array}$ & $\begin{array}{l}\text { Qualitative variables related environmental activities and } \\
\text { performance (as perception of respondents) } \\
\text { (called "environmental performance") }\end{array}$ & $\begin{array}{l}\text { Qualitative variables related competitive performance, } \\
\text { market performance image and reputation (as } \\
\text { perception of respondents) }\end{array}$ & $\begin{array}{l}\text { The development of environmentally friendly practices contributes to increase significantly } \\
\text { competitive performance in SMEs. }\end{array}$ \\
\hline $\begin{array}{l}\text { Misani and } \\
\text { Pogutz [103] }\end{array}$ & $\begin{array}{l}\text { Greenhouse gas emissions, Environmental management, Climate } \\
\text { change innovation (called "environmental performance") }\end{array}$ & $\begin{array}{l}\text { Tobin's q, Return on assets, Return on equity, Return on } \\
\text { sales, R \& D intensity (called "financial performance") }\end{array}$ & $\begin{array}{l}\text { Carbon performance improves financial performance up to a certain point, after which the } \\
\text { marginal benefits of further reduction of carbon emissions do not offset the marginal costs. }\end{array}$ \\
\hline \multirow{2}{*}{$\begin{array}{l}\text { Muhammad } \\
\text { et al. [104] }\end{array}$} & \multirow{2}{*}{ Toxicity risk score (called "corporate environmental performance") } & \multirow{2}{*}{$\begin{array}{l}\text { Firm market risk, Systematic risk, Downside risk (called } \\
\text { "company financial risk") }\end{array}$} & EP helps firms to reduce their financial risk. \\
\hline & & & EP is negatively associated with firm volatility and firm downside risk. \\
\hline \multirow{2}{*}{$\begin{array}{l}\text { Muhammad } \\
\text { et al. [105] }\end{array}$} & \multirow{2}{*}{ Toxicity risk score (called "corporate environmental performance") } & \multirow{2}{*}{$\begin{array}{l}\text { Return on assets, Tobin's q (called } \\
\text { "corporate financial performance") }\end{array}$} & $\begin{array}{l}\text { A strong positive association between EP and BP is confirmed during } \\
\text { the pre-financial crisis period. }\end{array}$ \\
\hline & & & No relationship between $\mathrm{EP}$ and $\mathrm{BP}$ is demonstrated during the financial crisis period. \\
\hline \multirow{2}{*}{$\begin{array}{l}\text { Sánchez-Medina } \\
\text { et al. [106] }\end{array}$} & \multirow{2}{*}{$\begin{array}{l}\text { Water consumption, Energy consumption, Solid waste (called } \\
\text { "environmental performance) }\end{array}$} & \multirow{2}{*}{$\begin{array}{l}\text { Return on assets, Market competitiveness, Personal } \\
\text { satisfaction, Profits (called "economic performance") }\end{array}$} & There is a bidirectional influence between EP and BP. \\
\hline & & & $\begin{array}{l}\text { Environmental compliance and environmental innovation have a significant mediating role } \\
\text { in this relationship. }\end{array}$ \\
\hline \multirow{3}{*}{$\begin{array}{l}\text { Trumpp and } \\
\text { Guenther [71] }\end{array}$} & \multirow{3}{*}{$\begin{array}{l}\text { Carbon performance, Waste intensity (called corporate } \\
\text { environmental performance) }\end{array}$} & \multirow{3}{*}{$\begin{array}{l}\text { Profitability, Stack market performance (called "corporate } \\
\text { financial performance") }\end{array}$} & There is a non-linear, U-shaped, relationship between EP and BP. \\
\hline & & & $\begin{array}{l}\text { Company's profitability is influenced by carbon performance and waste intensity both in } \\
\text { manufacturing and service industries. }\end{array}$ \\
\hline & & & $\begin{array}{l}\text { Stock market performance is only affected by carbon performance } \\
\text { in manufacturing industries. }\end{array}$ \\
\hline
\end{tabular}


Regarding the countries (Table 2, second column), 24 papers presented research conducted in Europe, six papers in Asia, five papers in north central America, four papers in Oceania, only one paper focused on Africa, and seven papers analyzed the topic from a worldwide perspective. The industrial sectors analyzed (Table 2, third column) indicated that the scientific debate includes all of the industrial sectors, with both environmentally dirty and clean processes. Of the types of research (Table 2, fourth column), the most frequently used was the survey, conducted through a data analysis of databases ( $62 \%$ of selected papers) or a direct interview of companies ( $21 \%$ of selected papers). Fifteen percent of papers included a literature review, and only one paper uses a multiple case study. The samples of the companies considered were always large (Table 2, fifth column), which confirmed the relevance of the research results and conclusions for each paper.

The variables considered by the authors to measure firms' environmental performance and business performance were very different (Table 3 , second and third columns). This lack of homogeneity was not always justified by different research goals or different research methodologies. For example, Eanhart and Lizal [72], Galdeano-Gómez et al. [74] and Yamaguchi and van Kooten [75], although they had similar research goals, employed different environmental and/or economic indicators to measure corporate performance. This was also true for the following studies: Menguc et al. [79], Molina-Azorin et al. [50], Yu et al. [81], López-Gamero et al. [82], Horvathova [89], Perez-Calderon et al. [91], Fujii et al. [63], De Burgos-Jimenez et al. [96], Qi et al. [100], and Sánchez-Medina et al. [106]. Moreover, this unjustified difference of performance variables was also present in studies with specific goals, such as papers with a greenhouse gas focus (see Dragomir [94], Wang et al. [101], Misani and Pogutz [103], and Trumpp and Guenther 2015 [71]) or papers that focused on a financial crisis period (see Gallego-Alvarez et al. [97], Muhammad et al. [104], Muhammad et al. [105], and Trumpp and Guenther [71]). Furthermore, with reference of specific industrial sectors analyzed (Table 3 , third column), we recognize a non-homogeneity of variables considered to measure EP and BP (Table 3, second and third columns): e.g., López-Gamero et al. [77], Molina-Azorín et al. [50], and Trumpp and Guenther [71] in services sector; Zeng et al. [84], Figge and Hahn [70], Fuji et al. [63], and Sánchez-Medina et al. [106] in manufacturing sector; Kranjc et al. [73], and Galdeano-Gómez [74] in food sector.

The conclusions of the papers (Table 3, fourth column) were often different: some authors explained the direction of the EP-BP relationship (26 papers); some authors confirmed or denied an EP-BP relationship but did not explicitly describe a direction (15 papers); some authors stated other propositions about corporate environmental and business strategies (nine papers); and some authors demonstrated variables that affected the EP-BP relationship (16 papers). Almost all of the authors conducted statistical analyses to verify the correlation and declared the robustness of their research results.

\subsection{Determining Whether There Is a Relationship between EP-BP of Enterprises in the Selected Papers (Step III)}

The third step (conducted as specified in Table 1) was to confirm or deny the first two research hypotheses, $\mathrm{H} 0$ or H1. The second and third columns in Table 4 present a synthesis of these results.

Almost all of the authors found a relationship between EP and BP and broadly confirmed hypothesis H0, "There is a relationship between EP and BP".

However, in some cases, the relationship was weak (Iraldo et al. [76], Yu et al. [81], and Horvathova [89]) or was limited to specific variables or conditions (Horvathova [51], Lopez-Gamero et al. [82], Muhammad et al. [105], and Trumpp and Guenther 2015 [71]). 
Table 4. Analyses of the papers concerning EP-BP relationship of enterprises with the research hypotheses.

\begin{tabular}{|c|c|c|c|c|c|c|c|}
\hline Paper & Ho * & H1 * & H0.1 ** & $\mathrm{H} 0.2$ ** & $\mathrm{H} 0.3^{* *}$ & Ho. $4^{* *}$ & Ho.x ** \\
\hline Wagner [25] & True & False & $\begin{array}{l}\text { True for firms with a } \\
\text { pollution prevention } \\
\text { strategy } \\
\text { False for firms with } \\
\text { end-of-pipe strategy }\end{array}$ & Not analyzed & Not analyzed & Not analyzed & Not analyzed \\
\hline Earnhart and Lizal [72] & True & False & Not analyzed & Not analyzed & True & True & Not analyzed \\
\hline Kranjc et al. [73] & True & False & Not analyzed & Not analyzed & Not analyzed & Not analyzed & Not analyzed \\
\hline Galdeano-Gómez [74] & True & False & True & Not analyzed & True & Not analyzed & Not analyzed \\
\hline $\begin{array}{l}\text { Yamaguchi and } \\
\text { van Kooten [75] }\end{array}$ & True & False & Not strongly supported & Not analyzed & True & Not analyzed & $\begin{array}{l}\text { Inverted U-shaped } \\
\text { hypothesis partially true }\end{array}$ \\
\hline Boons and Wagner [37] & True & False & $\begin{array}{l}\text { True at the market level. } \\
\text { Not strongly supported at } \\
\text { the firm level }\end{array}$ & Not analyzed & $\begin{array}{l}\text { True at the market level. } \\
\text { Not strongly supported at } \\
\text { the firm level }\end{array}$ & Not analyzed & Not analyzed \\
\hline Iraldo et al. [76] & Not strongly supported & Not strongly supported & False & Not analyzed & Not analyzed & Not analyzed & Not analyzed \\
\hline $\begin{array}{l}\text { López-Gamero } \\
\text { et al. [77] }\end{array}$ & True & False & $\begin{array}{l}\text { True with mediator } \\
\text { variables }\end{array}$ & Not analyzed & True as mediator variable & Not analyzed & $\begin{array}{l}\text { Mediator variables: firm's } \\
\text { resources and competitive } \\
\text { advantage }\end{array}$ \\
\hline $\begin{array}{l}\text { Mazzanti and } \\
\text { Zoboli [78] }\end{array}$ & True & False & True & Not analyzed & True & Not analyzed & $\begin{array}{l}\text { Inverted U-shaped } \\
\text { hypothesis partially true }\end{array}$ \\
\hline Menguc et al. [79] & True & False & True & Not analyzed & Not analyzed & Not analyzed & $\begin{array}{l}\text { Mediator variables: } \\
\text { government regulation and } \\
\text { consumer sensitivity }\end{array}$ \\
\hline $\begin{array}{l}\text { Molina-Azorín } \\
\text { et al. [80] }\end{array}$ & True & False & True & Not analyzed & True & Not analyzed & Not analyzed \\
\hline $\begin{array}{l}\text { Molina-Azorín } \\
\text { et al. [50] }\end{array}$ & True & False & True & Not analyzed & Not analyzed & Not analyzed & Not analyzed \\
\hline Yu et al. [81] & Not strongly supported & Not strongly supported & False & Not analyzed & Not analyzed & Not analyzed & Not analyzed \\
\hline Horváthová [51] & $\begin{array}{l}\text { True in common law } \\
\text { countries }\end{array}$ & Not strongly supported & $\begin{array}{l}\text { True in common law } \\
\text { countries }\end{array}$ & Not analyzed & $\begin{array}{l}\text { True in common law } \\
\text { countries }\end{array}$ & Not analyzed & Not analyzed \\
\hline $\begin{array}{l}\text { López-Gamero } \\
\text { et al. [82] }\end{array}$ & $\begin{array}{l}\text { True in voluntary } \\
\text { approach } \\
\text { False in } \\
\text { command-and-control } \\
\text { approach }\end{array}$ & $\begin{array}{l}\text { False in voluntary } \\
\text { approach } \\
\text { True in } \\
\text { command-and-control } \\
\text { approach }\end{array}$ & True & Not analyzed & True & Not analyzed & Not analyzed \\
\hline Wagner [83] & True & False & True & Not analyzed & Not analyzed & Not analyzed & Not analyzed \\
\hline Zeng et al. [84] & True & False & True & Not analyzed & Not analyzed & Not analyzed & Not analyzed \\
\hline $\begin{array}{l}\text { Heras-Saizarbotoria } \\
\text { et al. [85] }\end{array}$ & True & False & False & True & Not analyzed & Not analyzed & Not analyzed \\
\hline
\end{tabular}


Table 4. Cont.

\begin{tabular}{|c|c|c|c|c|c|c|c|}
\hline Paper & Ho * & H1 * & $\mathrm{H} 0.1^{* *}$ & $\mathrm{H} 0.2 * *$ & $\mathrm{H} 0.3^{* *}$ & $\mathrm{H} 0.4^{* *}$ & H0. $x^{* *}$ \\
\hline Iwata and Okada [86] & True & False & $\begin{array}{l}\text { True in clean industries and } \\
\text { on GHG reduction. } \\
\text { False in dirty industries and } \\
\text { on waste reduction }\end{array}$ & Not analyzed & Not analyzed & Not analyzed & $\begin{array}{l}\text { Mediator variables: clean or } \\
\text { dirty industry, firm growth } \\
\text { rate }\end{array}$ \\
\hline Salama et al. [87] & True & False & True & Not analyzed & True & Not analyzed & Not analyzed \\
\hline Zeng et al. [88] & True & False & $\begin{array}{l}\text { True for some variables. } \\
\text { False for some other } \\
\text { variables }\end{array}$ & Not analyzed & $\begin{array}{l}\text { True for some variables } \\
\text { False for some other } \\
\text { variables }\end{array}$ & Not analyzed & $\begin{array}{l}\text { Mediator variables: } \\
\text { non-financial indexes } \\
\text { related economic } \\
\text { performance }\end{array}$ \\
\hline Figge and Hahn [70] & True & False & True & Not analyzed & True & Not analyzed & $\begin{array}{l}\text { Cyclic hypothesis: } \\
\text { H0.3-H0.1-H0.3 }\end{array}$ \\
\hline Horváthová [89] & Not strongly supported & Not strongly supported & $\begin{array}{l}\text { True in long-term. } \\
\text { False in a short-term }\end{array}$ & Not analyzed & Not analyzed & Not analyzed & $\begin{array}{l}\text { Mediator variable: } \\
\text { time horizon }\end{array}$ \\
\hline Lioui and Sharma [90] & True & False & $\begin{array}{l}\text { True in indirect BP. } \\
\text { False in direct financial } \\
\text { performance }\end{array}$ & Not analyzed & Not analyzed & Not analyzed & $\begin{array}{l}\text { Mediator variables: direct } \\
\text { vs. indirect BP }\end{array}$ \\
\hline $\begin{array}{l}\text { Perez-Calderon } \\
\text { et al. [91] }\end{array}$ & True & False & $\begin{array}{l}\text { True in environmentally } \\
\text { sensitive companies. } \\
\text { False in not environmentally } \\
\text { sensitive companies }\end{array}$ & Not analyzed & True & Not analyzed & $\begin{array}{l}\text { Mediator variables: } \\
\text { industrial sector, BP indexes, } \\
\text { EP indexes, regions, } \\
\text { time-period }\end{array}$ \\
\hline Tang et al. [92] & True & False & True & Not analyzed & Not analyzed & Not analyzed & $\begin{array}{l}\text { Mediator variables: } \\
\text { customer satisfaction and } \\
\text { corporate reputation }\end{array}$ \\
\hline Albertini [54] & True & False & True & Not analyzed & Not analyzed & Not analyzed & $\begin{array}{l}\text { Mediator variables: } \\
\text { industrial sector, BP indexes, } \\
\text { EP indexes, regions, } \\
\text { time-period }\end{array}$ \\
\hline $\begin{array}{l}\text { Apeaning and } \\
\text { Thollander [93] }\end{array}$ & True & False & Not analyzed & Not analyzed & True & True & $\begin{array}{l}\text { Bi-directional hypothesis: } \\
\text { H0.1 and H0.3 are true at the } \\
\text { same time }\end{array}$ \\
\hline Dixon-Fowler et al. [52] & True & False & True & Not analyzed & True & Not analyzed & $\begin{array}{l}\text { Mediator variables: BP } \\
\text { indexes, regions, } \\
\text { institutional vs. } \\
\text { self-reported data. }\end{array}$ \\
\hline Dragomir [94] & True & False & Not strongly supported & Not analyzed & Not strongly supported & Not analyzed & $\begin{array}{l}\text { Neutral hypothesis: better } \\
\text { EP determines better BP but } \\
\text { also increases costs }\end{array}$ \\
\hline
\end{tabular}


Table 4. Cont

\begin{tabular}{|c|c|c|c|c|c|c|c|}
\hline Paper & $\mathrm{H}^{*}$ * & H1 * & H0.1 ** & $\mathrm{H} 0.2 * *$ & $\mathrm{H} 0.3^{* *}$ & H0.4** & Ho. $x^{* *}$ \\
\hline Fujii et al. [63] & True & False & $\begin{array}{l}\text { True in the case of } \mathrm{CO}_{2} \\
\text { emissions reduction }\end{array}$ & Not analyzed & Not analyzed & Not analyzed & $\begin{array}{l}\text { Inverted U-shaped } \\
\text { hypothesis partially true }\end{array}$ \\
\hline Youn et al. [95] & True & False & True & True & True & Not analyzed & Not analyzed \\
\hline $\begin{array}{l}\text { De Burgos-Jiménez et } \\
\text { al. [96] }\end{array}$ & True & False & True & Not analyzed & True & Not analyzed & Not analyzed \\
\hline Endrikat et al. [56] & True & False & True & Not analyzed & True & Not analyzed & $\begin{array}{l}\text { Bi-directional hypothesis: } \\
\text { H0. } 1 \text { and H0.3 are true at the } \\
\text { same time }\end{array}$ \\
\hline $\begin{array}{l}\text { Gallego-Álvarez } \\
\text { et al. [97] }\end{array}$ & True & False & True & Not analyzed & True & Not analyzed & $\begin{array}{l}\text { Cyclic hypothesis: } \\
\text { H0.3-H0.1-H0.3 }\end{array}$ \\
\hline Gotschol et al. [64] & True & False & True in the long run & Not analyzed & True also in the short run & Not analyzed & Not analyzed \\
\hline $\begin{array}{l}\text { Guenther and } \\
\text { Hoppe [53] }\end{array}$ & True & False & True & True & True & True & Not analyzed \\
\hline Moon et al. [98] & True & False & True & True & True & Not analyzed & Not analyzed \\
\hline Ortas et al. [99] & True & False & $\begin{array}{l}\text { True during bull markets. } \\
\text { False during the financial } \\
\text { crisis }\end{array}$ & Not analyzed & True & $\begin{array}{l}\text { True during } \\
\text { the financial } \\
\text { crisis }\end{array}$ & Not analyzed \\
\hline Qi et al. [100] & True & False & True & Not analyzed & True & Not analyzed & $\begin{array}{l}\text { Cyclic hypothesis: } \\
\text { H0.3-H0.1-H0.3 Moderator } \\
\text { variable: available of slack } \\
\text { resources }\end{array}$ \\
\hline Wang et al. [101] & True & False & True in all industrial sectors & Not analyzed & Not analyzed & Not analyzed & Not analyzed \\
\hline Larrán Jorge et al. [102] & True & False & True & Not analyzed & Not analyzed & Not analyzed & $\begin{array}{l}\text { Mediator variables: image } \\
\text { and relational marketing }\end{array}$ \\
\hline $\begin{array}{l}\text { Misani and } \\
\text { Pogutz [103] }\end{array}$ & True & False & True & Not analyzed & True & Not analyzed & $\begin{array}{l}\text { Inverted U-shaped } \\
\text { hypothesis true }\end{array}$ \\
\hline Muhammad et al. [104] & True & False & True & Not analyzed & Not analyzed & Not analyzed & $\begin{array}{l}\text { Moderator factors: available } \\
\text { technologies and } \\
\text { environmental regulation }\end{array}$ \\
\hline Muhammad et al. [105] & $\begin{array}{l}\text { True during the } \\
\text { pre-financial crisis } \\
\text { period. } \\
\text { Not true during the } \\
\text { financial crisis period }\end{array}$ & $\begin{array}{l}\text { False during the } \\
\text { pre-financial crisis } \\
\text { period. } \\
\text { Not false during the } \\
\text { financial crisis period }\end{array}$ & $\begin{array}{l}\text { True during the } \\
\text { pre-financial crisis period. } \\
\text { False during the financial } \\
\text { crisis period }\end{array}$ & Not analyzed & $\begin{array}{l}\text { True during the } \\
\text { pre-financial crisis period }\end{array}$ & $\begin{array}{l}\text { True during } \\
\text { the financial } \\
\text { crisis period }\end{array}$ & $\begin{array}{l}\text { Moderator variable: } \\
\text { available of slack resources }\end{array}$ \\
\hline $\begin{array}{l}\text { Sánchez-Medina } \\
\text { et al. [106] }\end{array}$ & True & False & True & Not analyzed & True & Not analyzed & $\begin{array}{l}\text { Mediating variables: } \\
\text { environmental compliance } \\
\text { and innovation }\end{array}$ \\
\hline $\begin{array}{l}\text { Trumpp and } \\
\text { Guenther [71] }\end{array}$ & True & False & $\begin{array}{l}\text { True for companies with } \\
\text { high EP }\end{array}$ & $\begin{array}{l}\text { True for companies } \\
\text { with low EP }\end{array}$ & Not analyzed & Not analyzed & U-shaped hypothesis true \\
\hline
\end{tabular}

${ }^{*} \mathrm{H} 0$ : There is a relationship between EP and BP; ${ }^{* *} \mathrm{H} 0.1$ : Better EP determines better BP; H0.2: Worse EP determines worse BP; H0.3: Better BP determines better EP; H0.4: Worse BP

determines worse EP; H0.x: There is another possible EP-BP relationship (e.g., inverse, non-linear, U-shaped, and conditional); H1: There is no relationship between EP and BP. 


\subsection{Determining the Type of EP-BP Relationship (Step IV)}

In the fourth step, we analyzed the 47 papers to confirm or deny the research sub-hypotheses, H0.1, H0.2, H0.3, H0.4 and H0.x. In Table 4, in columns four through eight, we show the results of this step.

Except for Kranjc et al. [73], all of the authors had at least one hypothesis about the direction of the relationship between EP and BP (i.e., the papers investigated not only hypotheses $\mathrm{H} 0$ and $\mathrm{H} 1$ but also one or more directional assumptions, such as sub-hypotheses H0.1, H.02, H.03, H.04 and/or H.0x.. Fourteen papers studied one single-directional hypothesis, while other papers had two or more directional hypotheses.

In almost all cases, the authors claimed to confirm their hypotheses, completely or partially, with the exception of two papers that did not confirm their hypotheses (Iraldo et al. [76], and Dragomir [94]).

The most frequently hypothesis analyzed and confirmed in the papers was the H0.1 hypothesis "Better EP determines better BP". Moreover, nine papers asserted that the H0.1 hypothesis was not true (or false) in specific conditions. The second most-confirmed hypothesis was the H0.3 hypothesis "Better BP determines better EP". Hypotheses H0.2 "Worse EP determines worse BP" and H0.4 "Worse $\mathrm{BP}$ determines worse $\mathrm{EP}^{\prime \prime}$ were claimed to be true in five papers.

As synthesized in the eighth column of Table 4 , more than $50 \%$ of the analyzed papers demonstrated other hypotheses about the direction in EP-BP relationship, as sub-hypothesis H0.x: starting from these papers we can underline some emerging threads.

Many authors demonstrated that the sub-hypotheses H0.1 and H0.3 were not necessary mutually exclusive: in two papers the "bi-directional hypothesis" H0.1-H0.3 is demonstrated; and three papers advanced a "cyclic hypothesis" in which better BP determined better EP that in turn determined better EP. These hypotheses may be considered to be an evolution of the "win-win approach" that was previously adopted by some authors to explain the positive two-way relationship between EP and BP $[30,107]$.

In four papers, the "inverted U-shaped hypothesis" was partially confirmed, highlighting how the EP-BP relationship is positive up to a maximum point and then becomes negative. This hypothesis is coherent with another theoretical model, named the "environmental Kuznet curve", at the firm level $[25,36]$. On the other hand, in one paper the "U-shaped hypothesis" was confirmed. However, one paper demonstrated a "neutral hypothesis", in which better EP determined better BP but at an additional cost.

Finally, 15 papers discussed various "conditional relationships", i.e., directions of the EP-BP relationship conditioned by "moderator" and/or "mediator" variables, that contribute to support or reduce the relationship under specific conditions (such as firms' size, Country or region, run-time, market competition, available technologies, internal control processes, innovation, environmental regulation, macro-economic conditions, corporate reputation and customer satisfaction).

\subsection{Analysing the Reasons for Any Disagreements in the Direction of EP-BP Relationship (Step V)}

In the fifth step, we reconsidered all the papers that concluded with disagreements in the direction of EP-BP, with the aim of underlining the possible reasons and adding further considerations to the discussion in Sections 4.2-4.4.

With reference to the summary of the papers in Table 2, the studies regard different industrial sectors (with both environmentally polluting and clean processes), and with different environmental strategies (with both environmental management tools and legal environmental requirements). This heterogeneity of companies' samples may have led to some inhomogeneity in the results. On the other hand, the high number of companies' samples analyzed and the research methodology adopted, as direct observations of public data or interviews, support the strength of the statistical results obtained in the studies.

With reference to the contents of the papers shown in Table 3, the main criticism that we can reveal is the extreme heterogeneity of environmental and economic variables considered in the 
studies. As discussed in Section 4.2, this inhomogeneity is generally not related to the industrial sector considered, neither to the Country nor to the research goals/topics. Even if it is possible to identify some "main" variables, which are adopted more frequently, especially with reference to the evaluation of BP, the great variety of variables selected by the authors is striking. Moreover, almost no author stated to refer to some international standards in defining the EP and BP measurement variables.

With reference to the analysis of the EP-BP relationship demonstrated by the papers, as shown in Table 4, the main disagreements concern some types of direction of this relationship, and, specifically, they indicate other possible EP-BP relationship, e.g., inverse, nonlinear, U-shaped, or conditioned by moderator factors. These results can be considered an evolution of the "Porter-Wagner dilemma", more than a lack of agreement among scholars.

\section{Discussion}

First, we discuss the results of sub-question (i): which recent papers address the relationship between the EP and BP of enterprises?

The relationship between EP and BP is a much-discussed and timely topic for the scientific community. From 2000 to 2015, 47 papers discussed this topic explicitly in different scientific journals, and nearly $50 \%$ of these papers were published in the last three years due to the financial crisis. The studies cover a range of industries and both developing and industrialized countries. The relationship between EP and BP has been studied both in large companies and in SMEs. The analyses were conducted primarily through surveys by statistical analysis of published data and through entrepreneurs' interviews.

A number of papers in recent years have discussed the relationship between environmental practices and firm performance in terms of environmental strategies, eco-design, innovation, and corporate social responsibility. In this paper, our focus was the relationship between EP and BP at the firm level, and for this reason, we excluded 182 papers: however it must be said that this abundance of articles on similar topics show that the link between environment and business is a "contentious issue" in scientific community [43], with great interest in many different fields.

Next, we discuss sub-question (ii): is there a sufficient consensus among authors to declare the existence of an EP-BP relationship?

All of the authors agree that there is a relationship between EP and BP; therefore, hypothesis H0 is confirmed. This agreement is notable because it includes different case studies in different countries and from different industrial sectors. However, some authors admit that this relationship is weak, at least for some variables.

Then, we consider sub-question (iii): is there a specific direction of the EP-BP relationship?

The two most studied sub-hypotheses are H0.1 "Better EP determines better BP" and H0.3 "Better BP determines better EP", corresponding to the "Porter-Wagner dilemma". These two hypotheses are also the most confirmed. However, several authors consider them to be complementary and not alternatives. Some authors indicate that hypothesis H0.1 is not confirmed and that hypothesis $\mathrm{H} 0.2$ "Worse EP determines worse BP" and H0.4 "Worse BP determines worse EP" are possible. The literature review revealed that other hypotheses have emerged to analyze the direction of the EP-BP relationship. The "cyclic hypothesis" and the "bi-directional hypothesis" confirm and extend the "win-win approach". The "inverted U-shaped hypothesis" proves to be an advanced solution in the decades-long debate. A "neutral hypothesis" remains valid in some studies.

Finally, we discuss the sub-question (iv): what are the reasons that do not lead the studies to a consensus view in the direction of EP-BP relationship?

First of all, we underline that in recent years there are new emergent hypotheses related to the EP-BP relationship, more complex and multi-factorial; however, the studies related to each emergent hypothesis are still few and for this reason in some cases they probably lead to different results. Therefore, we can confirm previous authors $[41,58,61]$ in the hypothesis that the methodological approaches to study this relationship are evolving, as "empirical data in search of a theory" [108]. 
On the other hand, there has been a significant evolution in research about the EP-BP relationship regarding the analysis of "mediators" and "moderators" variables, and the number of papers that consider the factors affecting this relationship has increased in recent years; however, the results of these studies are very different and often conflicting. Thus, the inconsistency, previously underlined by many authors (e.g., $[51,52,109,110])$, seems to have grown.

Beyond these considerations, it is necessary to recognize that the main criticism in the debate related to the "Porter-Wagner dilemma" concerns the extreme heterogeneity of the variables used by the authors to measure/verify the EP and BP. This lack of homogeneity generally is not linked by different research goals or different industrial sectors investigated, but it originates from different choices of the scientists. This problem has been partially highlighted in previous studies (e.g., $[19,32,84,86,89])$. It is certainly linked to the complexity of the topic, as well as been shown in $[2,38,43,48,66,111]$. However, it demonstrates that no consensus has yet been reached, despite numerous studies conducted in recent years [104,112]. Moreover, this inhomogeneity may be due to the fact that many studies related to the analysis of EP-BP relationship at the firms' level consider business cases, and consequently the choice of indicators for measuring EP and BP are affected by the availability and relevance of data for specific case studies analyzed. Then, being the business studies potentially very different among each other, it follows that the variables considered may be not comparable, and the conclusions may be not in agreement [113].

Overall, it is difficult to summarize the prevalent direction in the relationship between EP and $\mathrm{BP}$, not only because of the different results obtained in the testing of hypotheses but also because of the diversity of the variables considered in the studies. This gap represents a great disadvantage that still prevents scientists from supporting practitioners and enterprises in the strategic management of sustainability, through an agreement of comprehensive and effective assessment methods and tools [112-116]. Therefore, we remark that the priority of the scientific debate on "Porter-Wagner dilemma" is to share univocal methods to measure firms' performance. Coherently, it is important to share standardized information tools to support scientists, practitioners and managers in measuring the benefits of sustainable policies and practices [112,115,117].

\section{Conclusions and Future Perspectives}

In the debate regarding the business implications of environmental investment, the recent literature discusses the "Porter-Wagner dilemma": is the environment a "strategic competitive factor" or a "luxury good"? Our research aims to delve into the type of the relationship between EP and BP focusing in particular at the firm level. Through a systematic literature review of scientific papers published from 2000 to 2015, the research goals are: first, to determine what the prevalent cause and effect directions of EP-BP relationship are, and, secondly, to investigate the reasons for any disagreements in this topic among the scientists.

With this aim, two research hypotheses were elaborated to verify whether the EP-BP relationship exists, and five sub-hypotheses were expressed to investigate the potential directions of this relationship.

The results of our research confirm the relevance of this topic in different disciplines, with an increasing number of papers published in more recent years. Moreover, there is substantial commitment among the authors to demonstrate the existence of a relationship between the EP and the $\mathrm{BP}$ of companies in different countries and across industrial sectors. However, in some studies, this relationship is not verified for all of the environmental or economic variables considered.

In analyzing the direction of the EP-BP relationship, the two most confirmed sub-hypotheses are that "better EP determines better BP", as a "Porter point of view", and that "better BP determines better $\mathrm{EP}^{\prime}$, as a "Wagner point of view". These hypotheses resulted to be complementary and not alternative, as in a "bidirectional" or "cyclic" approach. Other hypotheses are also emerging, as "U-shaped", "inverted U-shaped", "neutral", and "conditional" relationship, sometimes with contradictory results. 
In discussing the reasons leading to disagreements among the scientists, we can remark that the debate on EP-BP relationship at the firms' level is very current today, with a continuous evolution in terms of types of relationships considered and variables adopted to measure the performance. However, we underline the urgency of standardization methods and tools to measure individual performance of companies, in order to enable the comparison of case studies results and to clearly lead the entrepreneurships in their efforts toward sustainable business.

The main limitations of this research concern the methodological choices adopted in the literature review. First, the sample of papers selected for the analysis is limited to the English papers publish in ISI journals, and does not consider other type of scientific documents, such as conference proceedings or books, and papers in non-English language. Second, our review includes papers considering all types of firms, both small/medium and large enterprises: it would be interesting to deepen the analysis of EP-BP relationship distinguishing based on the size of the firms, with the aim to confirm or deny the obtained results. Third, we have investigated exclusively the relationship between EP and BP, however, in recent years, the literature debate is also lively concerning corporate social responsibility (CSR), which includes both EP and BP performance: a relevant research perspective should consider the extension of the research to other CSR parameters.

From these results, we can derive some statements and future perspectives for managers, practitioners and scientists.

For managers, our study indicates that the relationship between EP and BP is bidirectional: consequently, for firms it is necessary to systematically consider the environmental implications of business strategies and vice versa, in a mutual positive correlation. This issue is so important that an understatement of this relationship may result in negative consequences for business performance.

For practitioners, we can affirm that a mutual negative correlation between EP and BP is possible, but the empirical evidences are still limited; therefore it is crucial to promote other studies in order to better define the mechanisms of the correlation between worse EP and worse BP.

For practitioners and scientists, other interesting types of relationship between EP and BP are emerging, more sophisticated and in line with the complexity of the topic. Thus, it is important to study in deep these hypotheses, e.g. combining U-shaped and inverted U-shaped models, and different mediator and moderator variables.

From this research, we can also underline an important lesson for the scientists: it is not possible to find an answer to the "Porter-Wagner dilemma" as long as the studies, even in their methodological rigor, lead to incomparable results because of the adoption of different indicators. In the future, collaboration between different disciplines and the sharing of comprehensive methods and tools to measure and relate to each other firms' EP and BP should be a priority to give clear and straightforward answers to enterprises, which are increasingly asking for evidence of the economic benefits associated with environmental commitment.

Author Contributions: This paper represents a result of teamwork. Anna Mazzi and Antonio Scipioni conceived and designed the research. Anna Mazzi performed the research, analyzed the data, discussed the results and wrote the paper. Sara Toniolo, Alessandro Manzardo and Jingzheng Ren supported in analyzing the data and writing the paper.

Conflicts of Interest: The authors declare no conflict of interest.

\section{References}

1. Chuang, S.P.; Huang, S.J. The effect of environmental corporate social responsibility on environmental performance and business competitiveness: The mediation of green information technology capital. J. Bus. Ethics 2016. [CrossRef]

2. Jabloński, A.; Jabloński, M. Research on business models in their life cycle. Sustainability 2016, 8, 430.

3. United Nations Environment Programme (UNEP). Towards a Green Economy: Pathways to Sustainable Development and Poverty Eradication; United Nations Environment Program: Nairobi, Kenya, 2011; ISBN: 978-92-807-3143-9. 
4. District Elderly Community Centre (DECC). The Energy Efficiency Strategy. The Energy Efficiency Opportunity in the UK; Department for Energy \& Climate Change (DECC): London, UK, 2012.

5. World Wide Fund for Nature (WWF). Living Planet Report 2014; Species and Spaces. People and Spaces; World Wildlife Fund: Gland, Switzerland, 2014.

6. OECD. The future of eco-innovation: The role of business models in green transformation. In Presented at the OECD/European Commission/Nordic Innovation Joint Workshop, Copenhagen, Denmark, 19-20 January 2012.

7. De Marchi, V.; Di Maria, E.; Micelli, S. Environmental strategies, upgrading and competitive advantage in global value chains. Bus. Strategy Environ. 2012, 22, 62-72. [CrossRef]

8. Kallis, G.; Gómez-Baggethun, E.; Zografos, C. To value or not to value? That is not the question. Ecol. Econ. 2013, 94, 97-105. [CrossRef]

9. Lisi, I.E. Translating environmental motivations into performance: The role of environmental performance measurement systems. Manag. Account. Res. 2015. [CrossRef]

10. Prakash, A. Why do firms adopt "beyond-compliance" environmental policies? Bus. Strategy Environ. 2001, 10, 286-299. [CrossRef]

11. Wagner, M. On the relationship between environmental management, environmental innovation and patenting: Evidence from German manufacturing firms. Res. Policy 2007, 36, 1578-1602. [CrossRef]

12. Chang, C.H. The influence of corporate environmental ethics on competitive advantage: The mediation role of green innovations. J. Bus. Ethics 2011, 104, 361-370. [CrossRef]

13. Chen, Y.S.; Chang, C.H.; Wu, F.S. Origins of green innovations: The differences between proactive and reactive green innovations. Manag. Decis. 2012, 50, 369-398. [CrossRef]

14. Dües, C.M.; Tan, K.H.; Lim, M. Green as the new Lean: How to use Lean practices as a catalyst to greening your supply chain. J. Clean. Prod. 2013, 40, 93-100. [CrossRef]

15. Lin, R.J.; Tan, K.H.; Geng, Y. Market demand, green product innovation, and firm performance: Evidence from Vietnam motorcycle industry. J. Clean. Prod. 2013, 40, 101-107. [CrossRef]

16. Roulet, T.; Touboul, S. The intentions with which the road is paved: Attitudes to liberalism as determinants of greenwashing. J. Bus. Ethics 2015, 128, 305-320. [CrossRef]

17. Salzmann, O.; Ionescu-Somers, A.; Steger, U. The business case for corporate sustainability: Literature review and research options. Eur. Manag. J. 2005, 23, 27-36. [CrossRef]

18. Khan, Z. Cleaner production: An ecological option for ISO certification in developing countries. J. Clean. Prod. 2008, 16, 22-27. [CrossRef]

19. Henri, J.F.; Journeault, M. Eco-control: The influence of management control systems on environmental and economic performance. Account. Organ. Soc. 2010, 35, 63-80. [CrossRef]

20. Lo, C.K.Y.; Yeung, A.C.L.; Cheng, T.C.E. The impact of environmental management systems on financial performance in fashion ad textile industries. Int. J. Prod. Econ. 2012, 135, 561-567. [CrossRef]

21. De Jong, P.; Paulraj, A.; Blome, C. The financial impact of ISO 14001 certification: Top-line, bottom-line, or both? J. Bus. Ethics 2014, 119, 131-149. [CrossRef]

22. Feng, T.; Wang, D. The influence of environmental management systems on financial performance: A moderated-mediation analysis. J. Bus. Ethics 2016, 135, 265-278. [CrossRef]

23. Walley, N.; Whitehead, B. It's not easy being green. Harv. Bus. Rev. 1994, 72, 132-163.

24. Boons, F. Greening products: A framework for product chain management. J. Clean. Prod. 2002, 10, 495-505. [CrossRef]

25. Wagner, M. How to reconcile environmental and economic performance to improve corporate sustainability: Corporate environmental strategies in the European paper industry. J. Environ. Manag. 2005, 76, 105-118. [CrossRef] [PubMed]

26. Wagner, M. Innovation and competitive advantages from the integration of strategic aspects with social and environmental management in European firms. Bus. Strategy Environ. 2008, 18, 291-306. [CrossRef]

27. Bhat, V.N. Does environmental compliance pay? Ecotoxicology 1998, 7, 221-225. [CrossRef]

28. Ederington, J.; Minier, J. Is environmental policy a secondary trade barrier? An empirical analysis. Can. J. Econ. 2003, 36, 137-154. [CrossRef]

29. Prakash, A.; Kollman, K. Policy modes, firms and the natural environment. Bus. Strategy Environ. 2004, 13, 107-128. [CrossRef] 
30. Cerin, P. Bringing economic opportunity into line with environmental influence: A discussion on the Coase theorem and the Porter and van der Linde Hypothesis. Ecol. Econ. 2006, 56, 209-225. [CrossRef]

31. Lee, K.H.; Kim, J.W. Integrating suppliers into green product innovation development: An empirical study in the semiconductor industry. Bus. Strategy Environ. 2011, 20, 527-538. [CrossRef]

32. Schneider, A.; Meins, E. Two dimensions of corporate sustainability assessment: Towards a comprehensive framework. Bus. Strategy Environ. 2013, 21, 211-222. [CrossRef]

33. Wunder, S. Revitising the concept of payments for environmental services. Ecol. Econ. 2015, 117, $234-243$. [CrossRef]

34. Porter, M.E. America's green strategy. Sci. Am. 1991, 264, 96.

35. Porter, M.E.; van der Linde, C. Toward a new concept of the environment-competitiveness relationship. J. Econ. Perspect. 1995, 9, 97-118. [CrossRef]

36. Hart, S.L.; Ahuja, G. Does it pay to be green? An empirical examination of the relationship between emission reduction and firm performance. Bus. Strategy Environ. 1996, 5, 30-37. [CrossRef]

37. Boons, F.; Wagner, M. Assessing the relationship between economic and ecological performance: Distinguishing system levels and the role of innovation. Ecol. Econ. 2009, 68, 1908-1914. [CrossRef]

38. Wagner, M.; Blom, J. The reciprocal and non-linear relationship of sustainability and financial performance. Bus. Ethics Eur. Rev. 2011, 20, 418-432. [CrossRef]

39. Jackson, L.; Singh, D. Environmental rankings and financial performance: An analysis of firms in the US food and beverage supply chain. Tour. Manag. Perspect. 2015, 14, 25-33. [CrossRef]

40. Lannelongue, G.; Gonzalez-Benito, J.; Gonzalez-Benito, O. Input, Output, and Environmental Management Productivity: Effects on Firm Performance. Bus. Strategy Environ. 2015, 24, 145-158. [CrossRef]

41. Wagner, M. The link of environmental and economic performance: Drivers and limitations of sustainability integration. J. Bus. Res. 2015, 68, 1306-1317. [CrossRef]

42. Albertini, E. What we know about environmental policy: An inductive typology of the research. Bus. Strategy Environ. 2015. [CrossRef]

43. Ramanathan, R. Understanding complexity: The curvilinear relationship between environmental performance and firm performance. J. Bus. Ethics 2016. [CrossRef]

44. Neuteleers, S.; Engelen, B. Talking money: How market-based valuation can determine environmental protection. Ecol. Econ. 2015, 117, 253-260. [CrossRef]

45. Siew, R.Y.J. A review of corporate sustainability reporting tools (SRTs). J. Environ. Manag. 2015, 164, $180-195$. [CrossRef] [PubMed]

46. Finster, M.P.; Hernke, M.T. Benefits organizations pursue when seeking competitive advantage by improving environmental performance. J. Ind. Ecol. 2014, 18, 652-662. [CrossRef]

47. Nicolăescu, E.; Alpopi, C.; Zaharia, C. Measuring Corporate Sustainability Performance. Sustainability 2015, 7, 851-865. [CrossRef]

48. Wood, D.J.; Jones, R.E. Stakeholder mismatching: A theoretical problem in empirical research on corporate social performance. Int. J. Organ. Anal. 1995, 3, 229-267. [CrossRef]

49. Fuchs, D.A.; Mazmanian, D. The greening of industry: Needs of the field. Bus. Strategy Environ. 1998, 7, 193-203. [CrossRef]

50. Molina-Azorín, J.F.; Claver-Cortés, E.; López-Gamero, M.D.; Tarí, J.J. Green management and financial performance: A literature review. Manag. Decis. 2009, 47, 1080-1100. [CrossRef]

51. Horváthová, E. Does environmental performance affect financial performance? A meta-analysis. Ecol. Econ. 2010, 70, 52-59. [CrossRef]

52. Dixon-Fowler, H.; Slater, D.; Johnson, J.L.; Ellstrand, A.E.; Romi, A.M. Beyond “does it pay to be green?" A meta-analysis of moderators of the CEP-CFP relationship. J. Bus. Ethics 2013, 112, 353-366. [CrossRef]

53. Guenther, E.; Hoppe, H. Merging limited perspectives. A synopsis of measurement approaches and theories of the relationship between corporate environmental and financial performance. J. Ind. Ecol. 2014, 18, 689-707. [CrossRef]

54. Albertini, E. Does environmental management improve financial performance? A meta-analytical review. Organ. Environ. 2013, 24, 431-457. [CrossRef]

55. Goyal, P.; Rahman, Z.; Kazmi, A.A. Corporate sustainability performance and firm performance research. Manag. Decis. 2013, 51, 361-379. [CrossRef] 
56. Endrikat, J.; Guenther, E.; Hoppe, H. Making sense of conflicting empirical findings: A meta-analytic review of the relationship between corporate environmental and financial performance. Eur. Manag. J. 2014, 32, 735-751. [CrossRef]

57. Lankoski, L. Corporate responsibility activities and economic performance: A theory of why and how they are connected. Bus. Strategy Environ. 2008, 17, 536-547. [CrossRef]

58. Potts, J.; Foster, J.; Straton, A. An entrepreneurial model of economic and environmental co-evolution. Ecol. Econ. 2010, 70, 375-383. [CrossRef]

59. Orlitzky, M. Institutional Logics in the Study of Organizations: The Social Construction of the Relationship between Corporate Social and Financial Performance. Bus. Ethics Q. 2011, 21, 409-444. [CrossRef]

60. Boons, F.; Montalvo, C.; Quist, J.; Wagner, M. Sustainable innovation, business models and economic performance: An overview. J. Clean. Prod. 2013, 45, 1-8. [CrossRef]

61. Van der Byl, C.A.; Slawinski, N. Embracing tensions in corporate sustainability. A review of research from win-wins and trade-offs to paradoxes and beyond. Organ. Environ. 2015, 28, 54-79. [CrossRef]

62. Figge, F.; Hahn, T. Value drivers of corporate eco-efficiency: Management accounting information for the efficient use of environmental resources. Manag. Account. Res. 2013, 24, 387-400. [CrossRef]

63. Fujii, H.; Iwata, K.; Kaneko, S.; Managi, S. Corporate environmental and economic performance of Japanese manufacturing firms: Empirical study for sustainable development. Bus. Strategy Environ. 2013, 22, 187-201. [CrossRef]

64. Gotschol, A.; de Giovanni, P.; Vinzi, V.E. Is environmental management and economically sustainable business? J. Environ. Manag. 2014, 144, 73-82. [CrossRef] [PubMed]

65. Luederitz, C.; Meyer, M.; Abson, D.J.; Gralla, F.; Lang, D.J.; Rau, A.L.; von Wehrden, H. Systematic student-driven literature review in sustainability science-An effective way to merge research and teaching. J. Clean. Prod. 2016, 119, 229-235. [CrossRef]

66. Van Beurden, P.; Gössling, T. The worth of values-A literature review on the relation between corporate social and financial performance. J. Bus. Ethics 2008, 7, 407-424. [CrossRef]

67. Mattioda, R.A.; Mazzi, A.; Canciglieri, O.; Scipioni, A. Determining the principal references of the social life cycle assessment of products. Int. J. Life Cycle Assess. 2015, 20, 1155-1165. [CrossRef]

68. Ambec, S.; Lanoie, P. Does it pay to be green? A systematic overview. Acad. Manag. Perspect. 2008, $22,45-62$.

69. Fifka, M.S. Corporate responsibility reporting and its determinants in comparative perspective-A review of the empirical literature and meta-analysis. Bus. Strategy Environ. 2013, 22, 1-35. [CrossRef]

70. Figge, F.; Hahn, T. Is green and profitable sustainable? Assessing the trade-off between economic and environmental aspects. Int. J. Prod. Econ. 2012, 140, 92-102. [CrossRef]

71. Trumpp, C.; Guenter, T. Too little or too much? Exploring U-shaped relationship between corporate environmental performance and corporate financial performance. Bus. Strategy Environ. 2015. [CrossRef]

72. Earnhart, D.; Lizal, L. Effects of ownership and financial performance on corporate environmental performance. J. Comp. Econ. 2006, 34, 111-129. [CrossRef]

73. Kranjc, D.; Mele, M.; Glavič, P. Improving the economic and environmental performances of the beet sugar industry in Slovenia: Increasing fuel efficiency and using by-products for ethanol. J. Clean. Prod. 2007, 15, 1240-1252.

74. Galdeano-Gómez, E. Does an endogenous relationship exist between environmental and economic performance? A resource-based view on the horticultural sector. Environ. Resour. Econ. 2008, 40, 73-89. [CrossRef]

75. Yamaguchi, J.; van Kooten, G.C. Do higher financial returns lead to better environmental performance in North America's forest products sector? Can. J. For. Res. 2008, 38, 2515-2525. [CrossRef]

76. Iraldo, F.; Testa, F.; Frey, M. Is an environmental management system able to influence environmental and competitive performance? The case of the eco-management and audit scheme (EMAS) in the European Union. J. Clean. Prod. 2009, 17, 1444-1452. [CrossRef]

77. López-Gamero, M.D.; Molina-Azorín, J.F.; Claver-Cortés, E. The whole relationship between environmental variables and firm performance: Competitive advantage and firm resources as mediator variables. J. Environ. Manag. 2009, 90, 3110-3121. [CrossRef] [PubMed]

78. Mazzanti, M.; Zoboli, R. Environmental efficiency and labour productivity: Trade-off or joint dynamics? A theoretical investigation and empirical evidence from Italy using NAMEA. Ecol. Econ. 2009, 68, 1182-1194. [CrossRef] 
79. Menguc, B.; Auh, S.; Ozanne, L. The interactive effect of internal and external factors on a proactive environmental strategy and its influence on a firm's performance. J. Bus. Ethics 2010, 94, 279-298. [CrossRef]

80. Molina-Azorín, J.F.; Claver-Cortés, E.; Pereira-Moliner, J.; Tarí, J.J. Environmental practices and firm performance: An empirical analysis in the Spanish hotel industry. J. Clean. Prod. 2009, 17, 516-524. [CrossRef]

81. Yu, V.; Ting, H.I.; Wu, Y.C.J. Assessing the greenness effort for European firms. Manag. Decis. 2009, 47, 1065-1079. [CrossRef]

82. López-Gamero, M.D.; Molina-Azorín, J.F.; Claver-Cortés, E. The potential of environmental regulation to change managerial perception, environmental management, competitiveness and financial performance. J. Clean. Prod. 2010, 18, 963-974. [CrossRef]

83. Wagner, M. The role of corporate sustainability performance for economic performance: A firm-level analysis of moderation effects. Ecol. Econ. 2010, 60, 1553-1560. [CrossRef]

84. Zeng, S.X.; Meng, X.H.; Yin, H.T.; Tam, V.W.Y.; Sun, L. Impact of cleaner production on business performance. J. Clean. Prod. 2010, 18, 975-983. [CrossRef]

85. Heras-Saizarbitoria, I.; Molina-Azorín, J.F.; Dick, G.P.M. ISO 14001 certification and financial performance: Selection-effect versus treatment-effect. J. Clean. Prod. 2011, 19, 1-12. [CrossRef]

86. Iwata, H.; Okada, K. How does environmental performance affect financial performance? Evidence from Japanese manufacturing firms. Ecol. Econ. 2011, 70, 1691-1700. [CrossRef]

87. Salama, A.; Anderson, K.; Toms, J.S. Does community and environmental responsibility affect firm risk? Evidence from UK panel data 1994-2006. Bus. Ethics Eur. Rev. 2011, 20, 192-204. [CrossRef]

88. Zeng, S.X.; Meng, X.H.; Zeng, R.C.; Tam, C.M.; Tam, V.W.Y.; Jin, T. How environmental management driving forces affect environmental and economic performance of SMEs: A study in the Northern China district. J. Clean. Prod. 2011, 19, 1426-1437. [CrossRef]

89. Horváthová, E. The impact of environmental performance on firm performance: Short-term costs and long-time benefits. Ecol. Indic. 2012, 84, 91-97. [CrossRef]

90. Lioui, A.; Sharma, Z. Environmental corporate social responsibility and financial performance: Disentangling direct and indirect effects. Ecol. Econ. 2012, 78, 100-111. [CrossRef]

91. Pérez-Calderón, E.; Milanés-Montero, P.; Ortega-Rossel, F.J. Environmental performance and firm value: Evidence from Dow Jones Sustainability Index Europe. Int. J. Environ. Res. 2012, 6, 1007-1014.

92. Tang, A.K.Y.; Lai, K.L.; Cheng, T.C.E. Environmental governance of enterprises and their economic upshot through corporate reputation and customer satisfaction. Bus. Strategy Environ. 2012, 21, 401-411. [CrossRef]

93. Apeaning, R.W.; Thollander, P. Barriers to and driving forces for industrial energy efficiency improvements in African industries-A case study of Ghana's largest industrial area. J. Clean. Prod. 2013, 53, $204-213$. [CrossRef]

94. Dragomir, V.D. Environmental performance and responsible corporate governance: An empirical note. Ekon Manag. 2013, 16, 33-51. [CrossRef]

95. Youn, S.; Yang, M.G.M.; Hong, P.; Park, K. Strategic supply chain partnership, environmental supply chain management practices, and performance outcomes: An empirical study of Korean firms. J. Clean. Prod. 2013, 56, 121-130. [CrossRef]

96. De Burgos-Jiménez, J.; Vázquez-Brust, D.; Plaza-Úbeda, J.A.; Dijkshoorn, J. Environmental protection and financial performance: An empirical analysis in Wales. Int. J. Oper. Prod. Manag. 2013, 33, 981-1018. [CrossRef]

97. Gallego-Álvarez, I.; García-Sánchez, I.M.; Vieira, C.D. Climate change and financial performance in times of crisis. Bus. Strategy Environ. 2014, 23, 361-374. [CrossRef]

98. Moon, S.; Bae, S.; Jeong, M.G. Corporate sustainability and economic performance: an empirical analysis of a voluntary environmental program in the USA. Bus. Strategy Environ. 2014, 23, 534-546. [CrossRef]

99. Ortas, E.; Moneva, J.M.; Álvarez, I. Sustainable supply chain and company performance: A global examination. Supply Chain Manag. 2014, 19, 332-350. [CrossRef]

100. Qi, G.Y.; Zeng, S.X.; Shi, J.J.; Meng, X.H.; Lin, H.; Yang, Q.X. Revisiting the relationship between environmental and financial performance in Chinese industry. J. Environ. Manag. 2014, 145, 349-356. [CrossRef] [PubMed]

101. Wang, L.; Li, S.; Gao, S. Do greenhouse gas emissions affect financial performance? An empirical examination of Australian public firms. Bus. Strategy Environ. 2014, 23, 505-519. [CrossRef] 
102. Larrán-Jorge, M.; Madueño, J.H.; Martínez-Martínez, D.; Sancho, M.P.L. Competitiveness and environmental performance in Spanish small and medium enterprises: Is there a direct link? J. Clean. Prod. 2015, 101, $26-37$. [CrossRef]

103. Misani, N.; Pogutz, S. Unraveling the effects of environmental outcomes and processes on financial performance: A non-linear approach. Ecol. Econ. 2015, 109, 150-160. [CrossRef]

104. Muhammad, N.; Scrimgeour, F.; Reddy, K.; Abidin, S. The impact of corporate environmental performance on market risk: The Australian industry case. J. Bus. Ethics 2015, 132, 347-362. [CrossRef]

105. Muhammad, N.; Scrimgeour, F.; Reddy, K.; Abidin, S. The relationship between environmental performance and financial performance in periods of growth and contraction: Evidence from Australian publicly listed companies. J. Clean. Prod. 2015, 102, 324-332. [CrossRef]

106. Sánchez-Medina, P.; Díaz-Pichardo, R.; Bautista-Cruz, A.; Toledo-López, A. Environmental compliance and economic and environmental performance: Evidence from handicrafts small businesses in Mexico. J. Bus. Ethics 2015, 126, 381-393. [CrossRef]

107. Porter, M.E.; Kramer, M. Creating shared value. How to reinvent capitalism and unleash the wave of innovation and growth. Harv. Bus. Rev. 2011, 89, 62-77.

108. Ullmann, A. Data in search of a theory: A critical examination of the relationship among social performance, social disclosure and economic performance of U.S. firms. Acad. Manag. Rev. 1985, 10, 540-557. [CrossRef]

109. Goll, I.; Rasheed, A.A. The moderating effect of environmental munificence and dynamism on the relationship between discretionary social responsibility and firm performance. J. Bus. Ethics 2004, 49, 41-54. [CrossRef]

110. Martínez-Ferrero, J.; Frías-Aceituno, J.V. Relationship between sustainable development and financial performance: International empirical research. Bus. Strategy Environ. 2015, 24, 20-39.

111. Lindhjem, H.; Hu, T.; Ma, Z.; Skjelvik, J.M.; Song, G.; Vennemo, H.; Wù, J.; Zhang, S. Environmental econominc impact assessment in China: Problems and prospects. Environ. Impact Assess. Rev. 2007, 27, 1-25. [CrossRef]

112. Coduras, A.; Saiz-Alvarez, J.M.; Ruiz, J. Measuring readlness for entrepreneurship: An information tool proposal. J. Innov. Knowl. 2016, 1, 99-108. [CrossRef]

113. Panwar, R.; Nybakk, E.; Hansen, E.; Pinkse, J. Does the business case matter? The effect of a perceived business case on small firms' social engagement. J. Bus. Ethics 2016. [CrossRef]

114. Cohen, B.; Smith, B.; Mitchell, R. Toward a sustainable conceptualization of dependent variables in entrepreneurship research. Bus. Strategy Environ. 2008, 17, 107-119. [CrossRef]

115. Searcy, C. Measuring enterprise sustainability. Bus. Strategy Environ. 2014. [CrossRef]

116. Trumpp, C.; Endrikat, J.; Zopf, C.; Guenther, E. Definition, conceptualization, and measurement of corporate environmental performance: A critical examination of a multidimensional construct. J. Bus. Ethics 2015, 126, 185-204. [CrossRef]

117. Wolf, S.; Schüyze, F.; Jaeger, C.C. Balance or synergies between environment and economy-A note on model structures. Sustainability 2016, 8, 761. [CrossRef]

(C) 2016 by the authors; licensee MDPI, Basel, Switzerland. This article is an open access article distributed under the terms and conditions of the Creative Commons Attribution (CC-BY) license (http://creativecommons.org/licenses/by/4.0/). 